\title{
Systematic Index
}

6. Family Chelisochidae BURR $\ldots \ldots \ldots \ldots \ldots \ldots \ldots \ldots \ldots \ldots \ldots \ldots$

1. Subfamily Genitalatinae SternmanN $\ldots \ldots \ldots \ldots \ldots \ldots \ldots \ldots \ldots, 2$

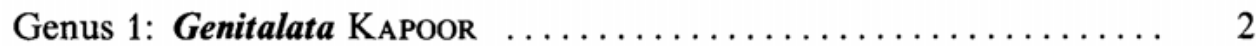

1. Genitalata mahajani KAPOOR $\ldots \ldots \ldots \ldots \ldots \ldots \ldots \ldots \ldots \ldots, 3$

2. Subfamily Chelisochellinae SteInMann $\ldots \ldots \ldots \ldots \ldots \ldots \ldots, 4$

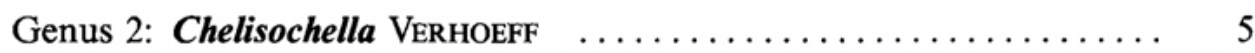

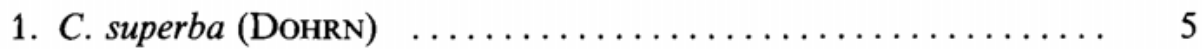

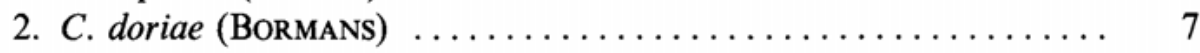

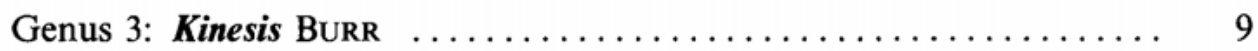

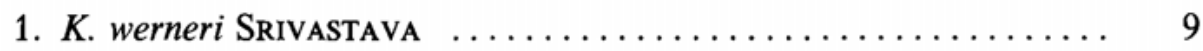

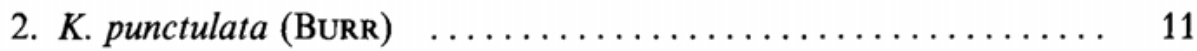

3. K. mounseyi BURR ............................ 12

4. K. significans sp. nov. ........................ 13

3. Subfamily Chelisochinae BurR $\ldots \ldots \ldots \ldots \ldots \ldots \ldots \ldots \ldots, 15$

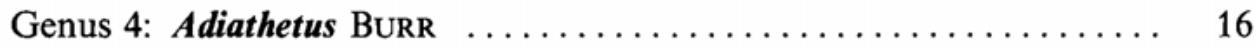

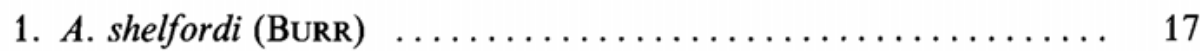

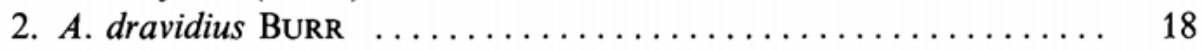

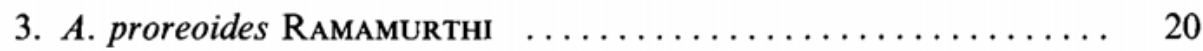

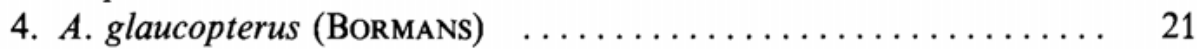

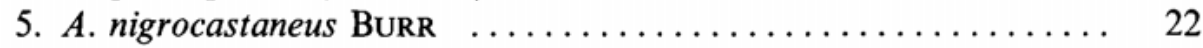

6. A. phoenix sp. nov. ............................ 23

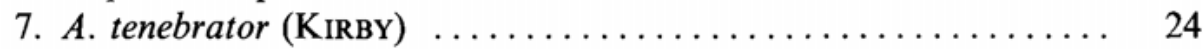

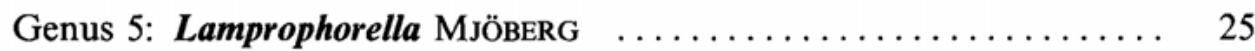

1. L. kervillei (BURR) .......................... 26

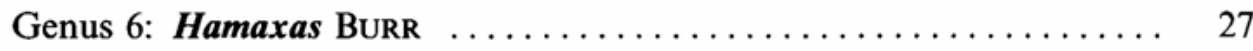

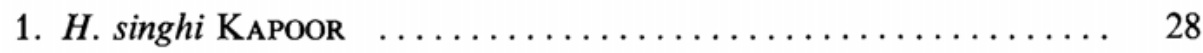




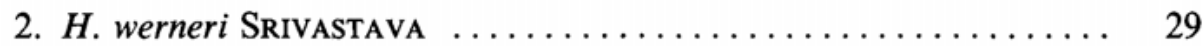

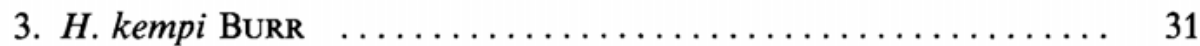

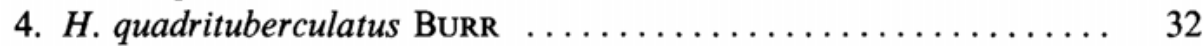

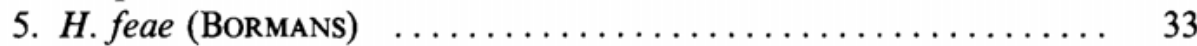

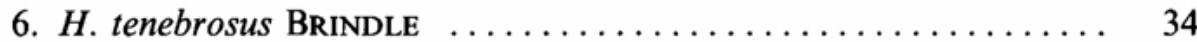

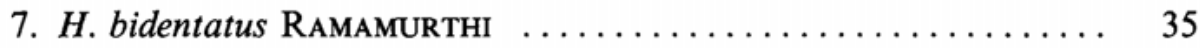

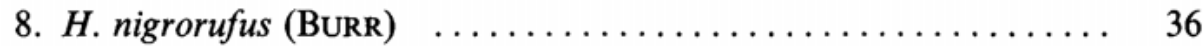

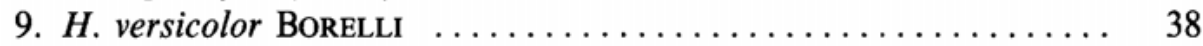

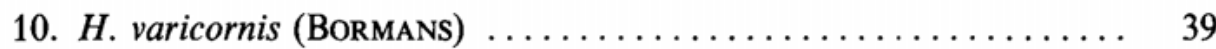

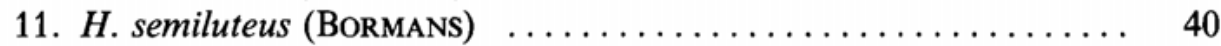

12. H. kozlovi Semenov \& Bey-BienKo $\ldots \ldots \ldots \ldots \ldots \ldots \ldots \ldots, 41$

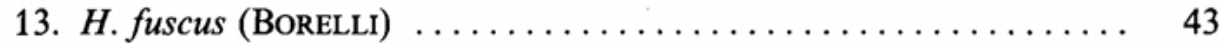

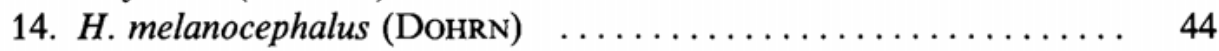

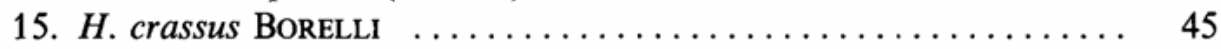

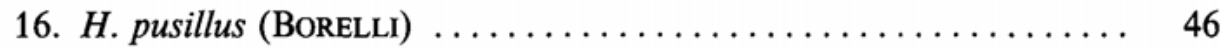

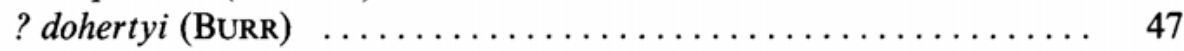

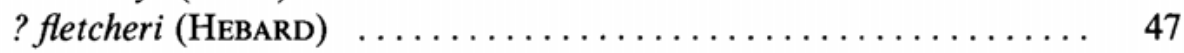

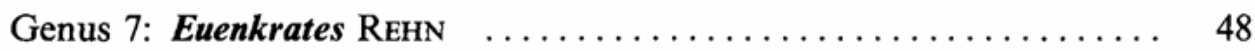

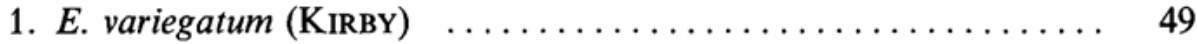

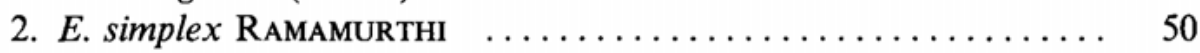

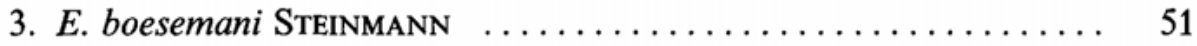

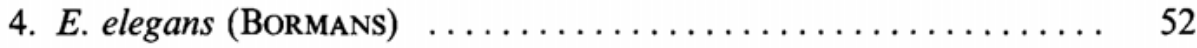

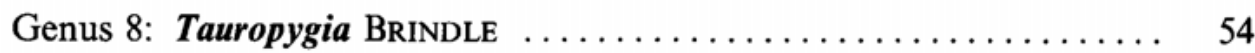

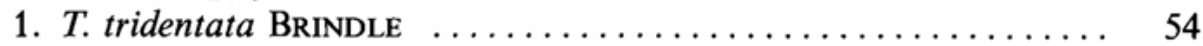

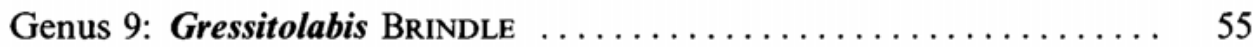

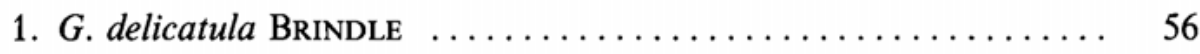

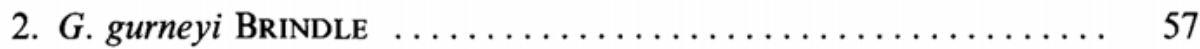

Genus 10: Adiathella Brindle $\ldots \ldots \ldots \ldots \ldots \ldots \ldots \ldots \ldots \ldots \ldots \ldots$

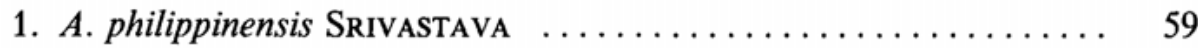

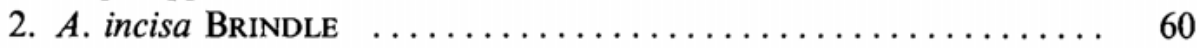

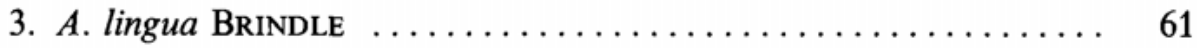

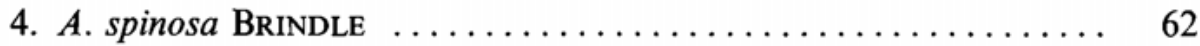

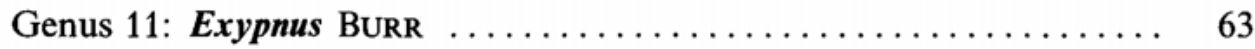

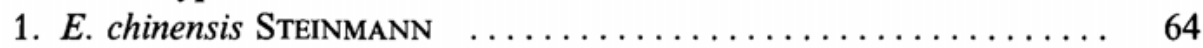

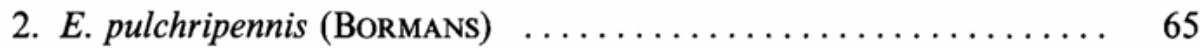

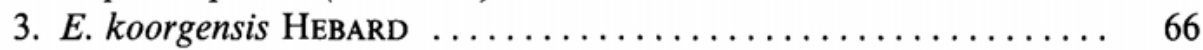


Genus 12: Schizochelisoches StEINMANN $\ldots \ldots \ldots \ldots \ldots \ldots \ldots \ldots 68$

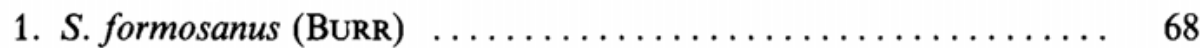

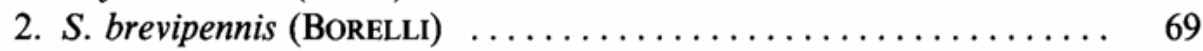

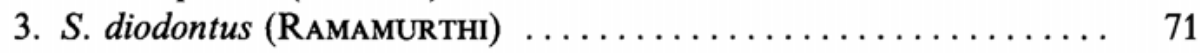

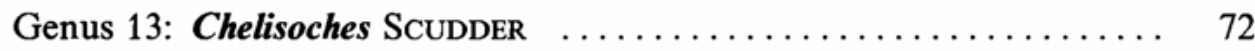

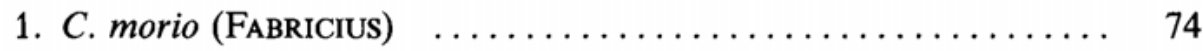

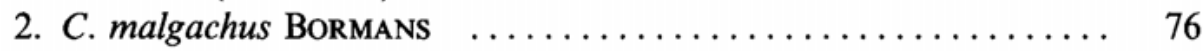

3. C. chopardi HincKs ............................. 77

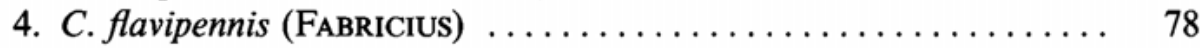

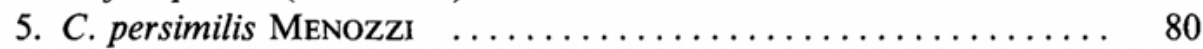

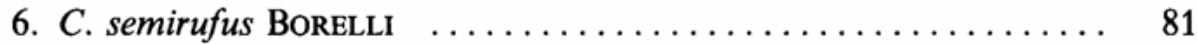

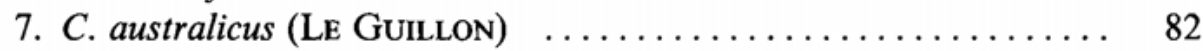

8. C. ater BoRMANS ........................... 83

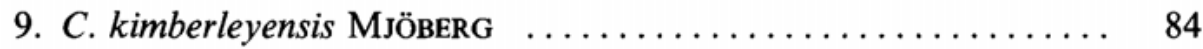

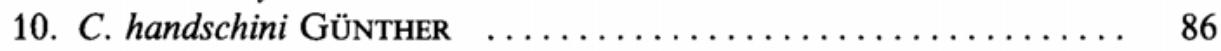

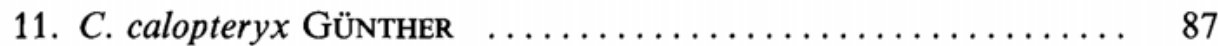

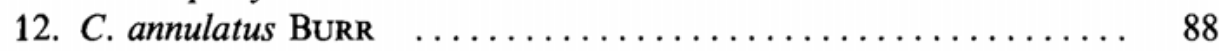

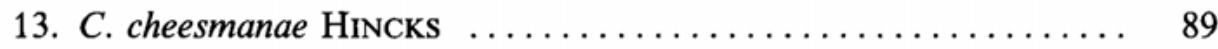

? paravicinii GÜNTHER $\ldots \ldots \ldots \ldots \ldots \ldots \ldots \ldots \ldots \ldots \ldots \ldots \ldots$

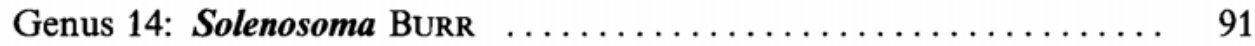

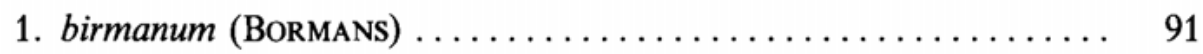

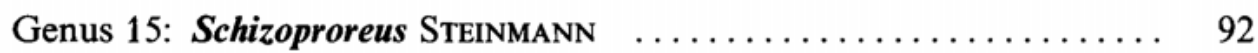

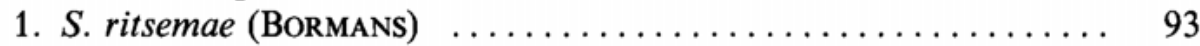

2. S. shaffii (BHARADWAJ \& KAPOOR) $\ldots \ldots \ldots \ldots \ldots \ldots \ldots \ldots . \ldots \ldots$

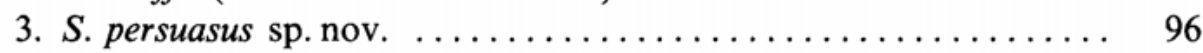

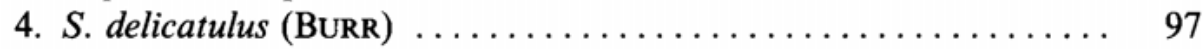

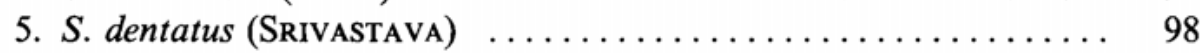

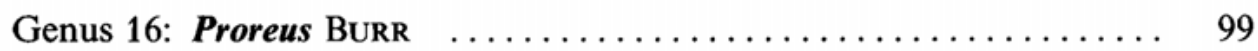

1. P. orpheus sp. nov. ............................ 101

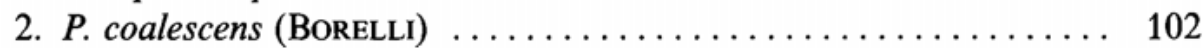

3. P. variopictus (Bormans) $\ldots \ldots \ldots \ldots \ldots \ldots \ldots \ldots \ldots \ldots \ldots$

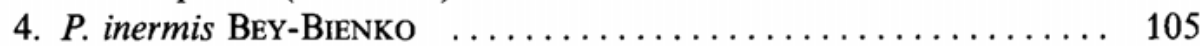

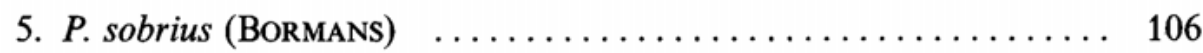

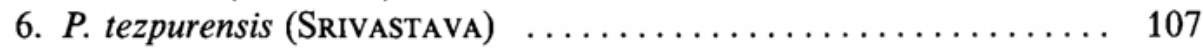

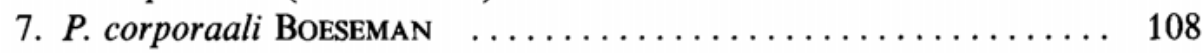

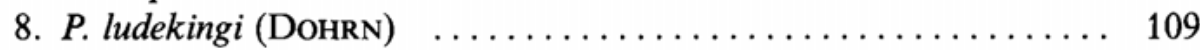

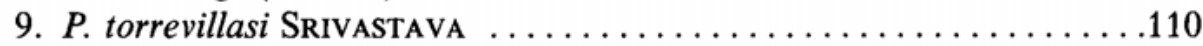

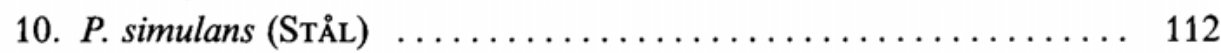




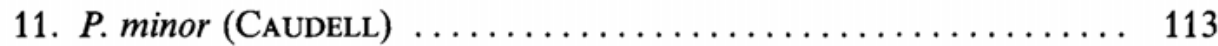

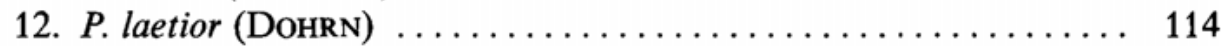

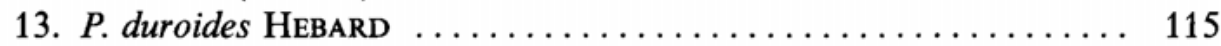

14. P. unidentatus Bey-BIEnKo ....................... 116

15. P. abdominalis RAMAMURTHI $\ldots \ldots \ldots \ldots \ldots \ldots \ldots \ldots \ldots \ldots \ldots \ldots$

16. P. cunctator BURR .............................. 118

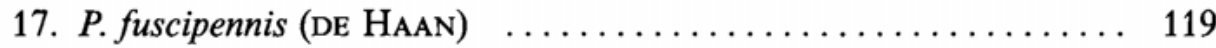

18. P. weissi (BURR) ................................ 120

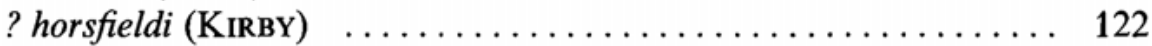

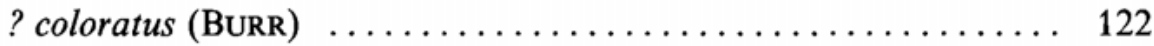

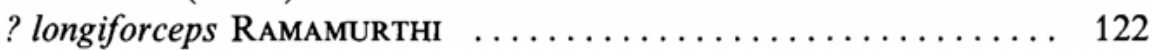

? ramamurthii KAPOOR ......................... 122

? chatterjeei KAPOOR, BHARADWAJ \& BeNERJEE ........... 122

? biratnagarensis KAPOOR, MALla \& SHAH .............. 122

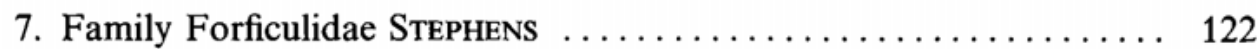

1. Subfamily Ancistrogastrinae VerHOEFF $\ldots \ldots \ldots \ldots \ldots \ldots \ldots \ldots \ldots$

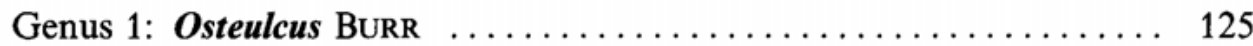

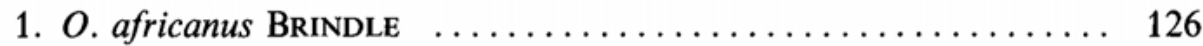

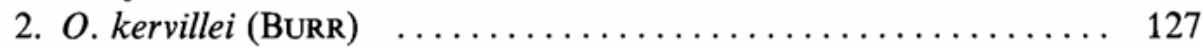

Genus 2: Litocosmia HeBARD $\ldots \ldots \ldots \ldots \ldots \ldots \ldots \ldots \ldots \ldots \ldots \ldots$

1. L. roraimae HEBARD .............................. 129

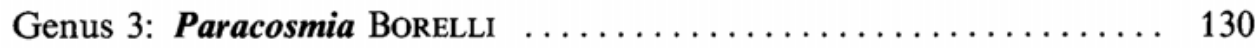

1. P. ambesa (MenOzzI) ......................... 130

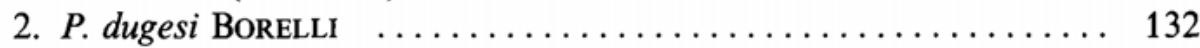

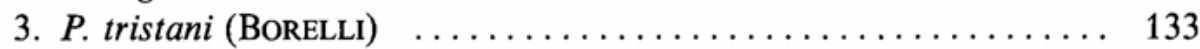

4. P. silvestrii BORELLI ........................... 134

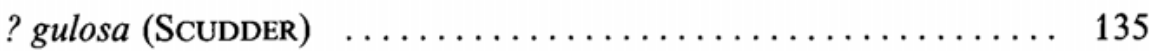

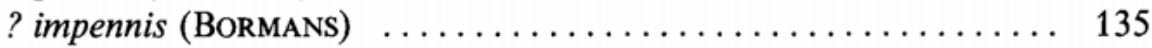

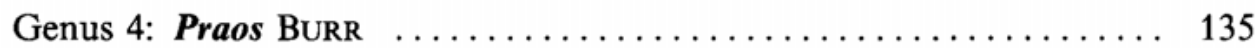

1. P. alfari (BoRELLI) ............................ 136

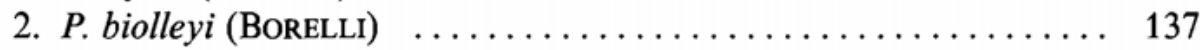

3. P. curiosus sp. nov. ........................... 139

4. P. perditus (BoRELLI) ........................... 140

5. P. robustus BoRELLI ........................... 141

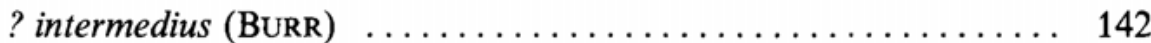

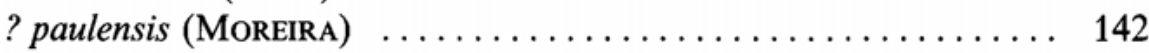




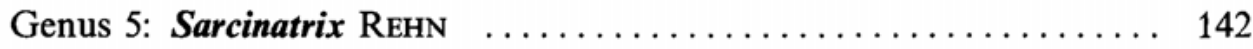

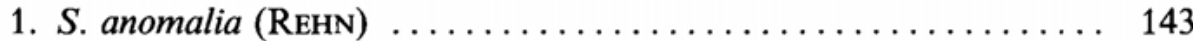

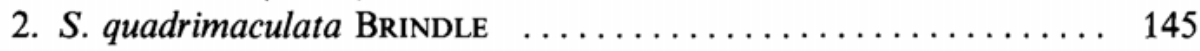

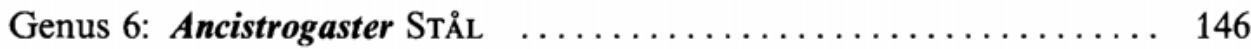

1. A. globalis sp. nov. ........................... 147

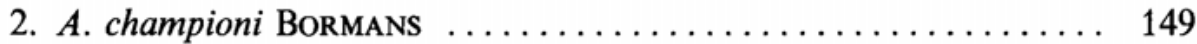

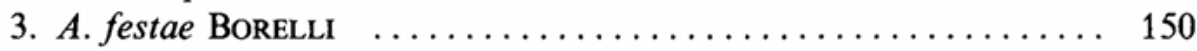

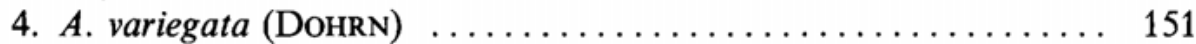

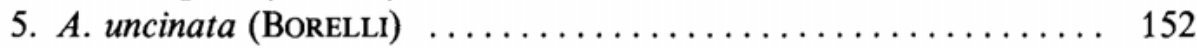

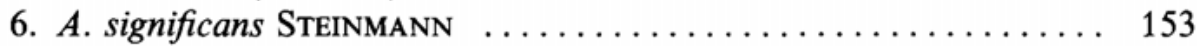

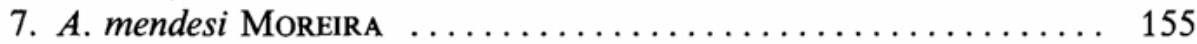

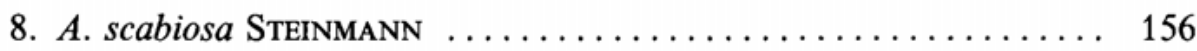

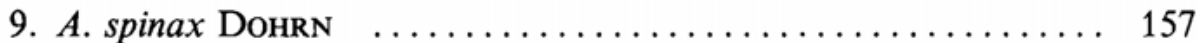

10. A. arthritica SCUDDER .......................... 158

11. A. maculifera (DoHRN) .......................... 159

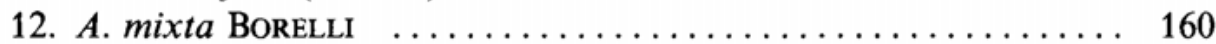

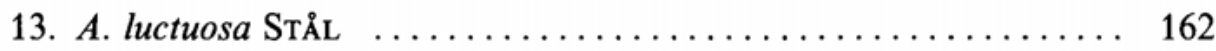

? burri KIRBY .............................. 163

2. Subfamily Cosmiellinae Steinmann $\ldots \ldots \ldots \ldots \ldots \ldots \ldots \ldots, 163$

Genus 7: Brachycosmiella gen. nov. $\ldots \ldots \ldots \ldots \ldots \ldots \ldots \ldots \ldots, 164$

1. B. sogai (BRINDLE) …........................... 165

Genus 8: Paracosmiella gen. nov. $\ldots \ldots \ldots \ldots \ldots \ldots \ldots \ldots \ldots \ldots$

1. P. simplex (BRINDLE) . .......................... 166

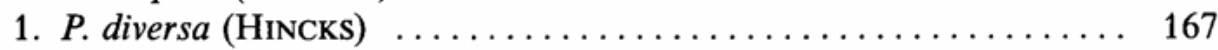

Genus 9: Forcepsia MoreIRA $\ldots \ldots \ldots \ldots \ldots \ldots \ldots \ldots \ldots \ldots \ldots$

1. F. pulla MoReIRA ............................... 169

Genus 10: Neocosmiella Hebard $\ldots \ldots \ldots \ldots \ldots \ldots \ldots \ldots \ldots \ldots \ldots$

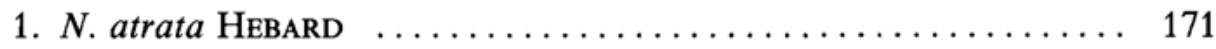

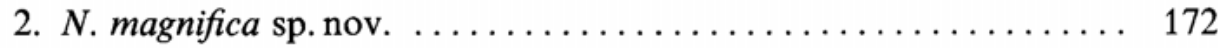

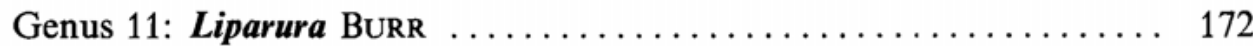

1. L. simplex BrindLE ............................ 173

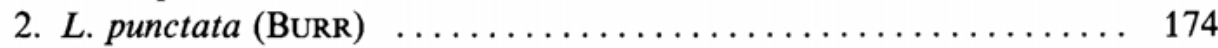

3. L. montuosa SteinMANN ......................... 176

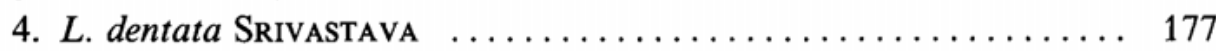

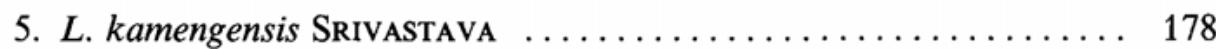

6. L. serrata SRIVASTAVA ......................... 179 


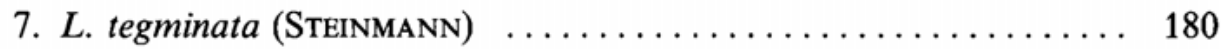

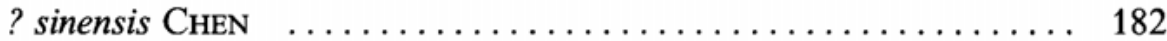

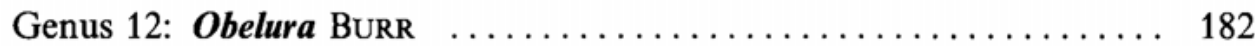

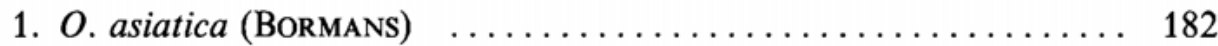

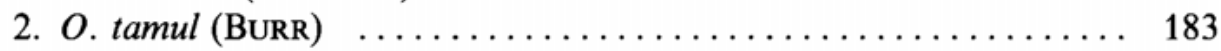

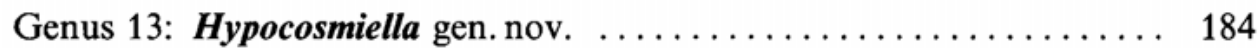

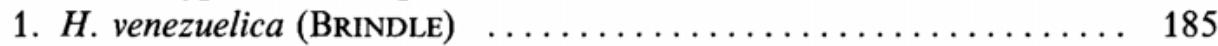

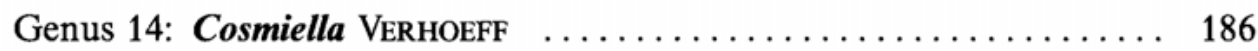

1. C. brasiliensis MoreIRA ....................... 188

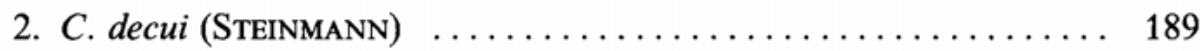

3. C. rebus (BURR) .............................. 190

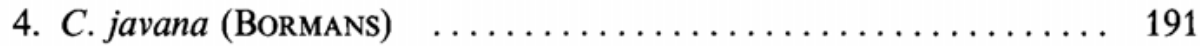

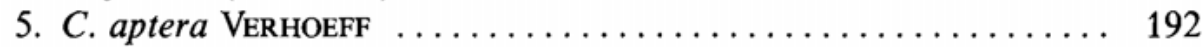

6. C. tuberculata (BoRELLI) ....................... 194

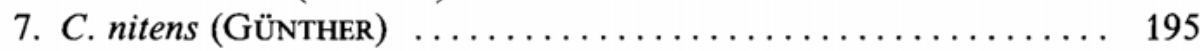

8. C. dubia (BoRMANs) ......................... 195

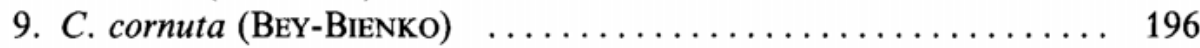

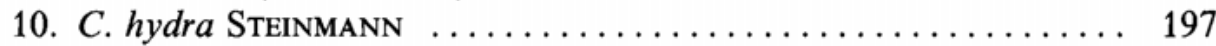

11. C. exornata SteInMANN ........................ 198

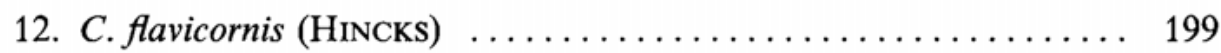

13. C. longiforceps (BRINDLE) ........................... 201

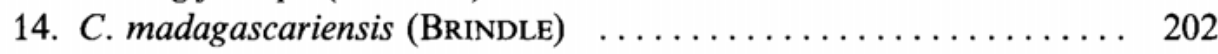

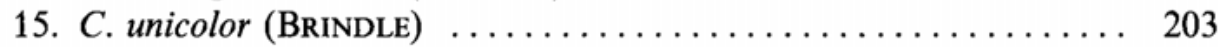

16. C. eridana SteinManN ........................... 204

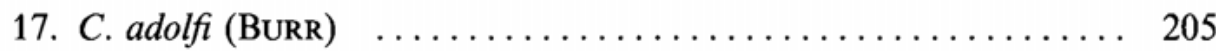

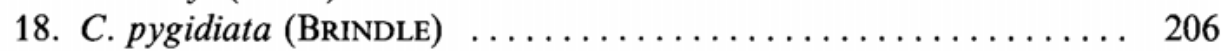

19. C. bilobata (BRINDLE) …...................... 207

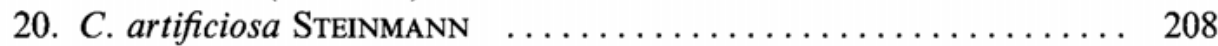

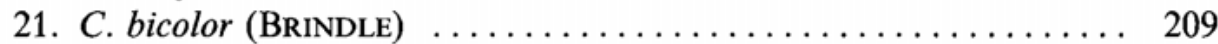

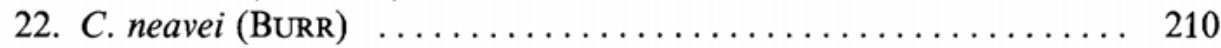

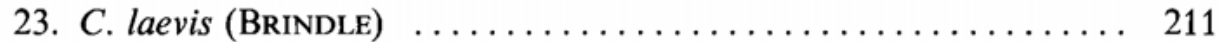

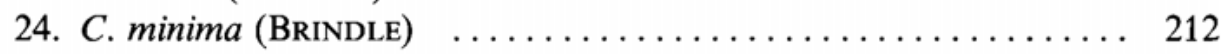

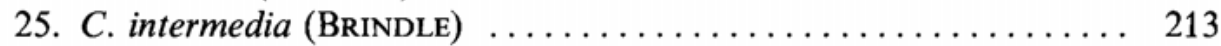

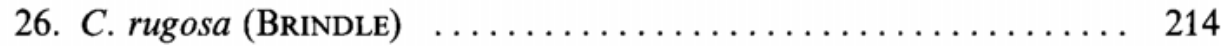

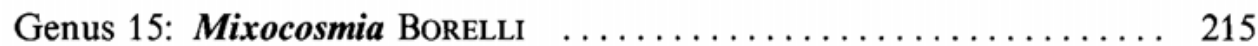

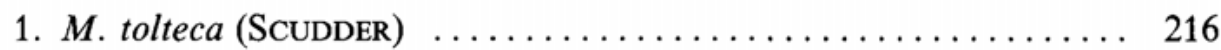

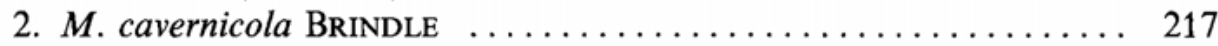

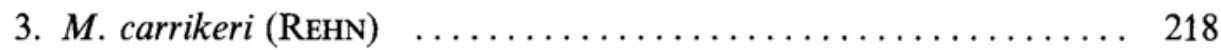




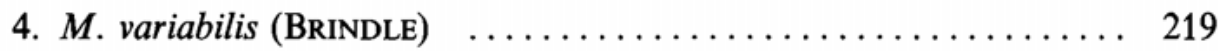

5. M. tristani BoRelLI ............................ 221

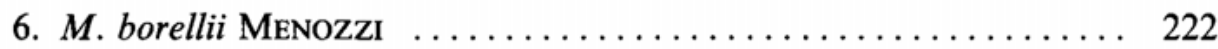

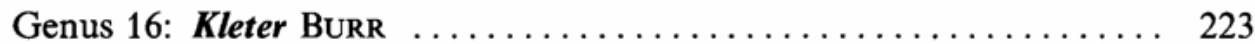

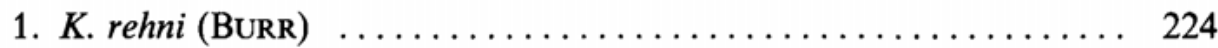

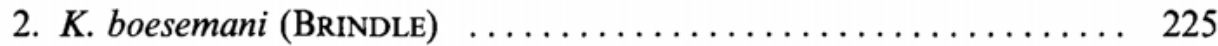

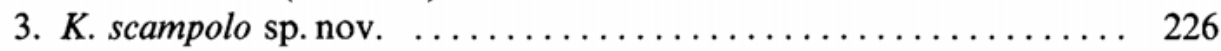

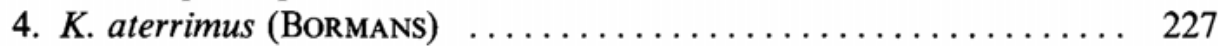

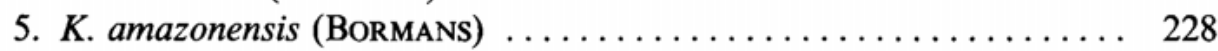

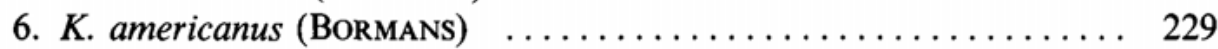

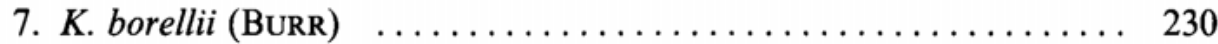

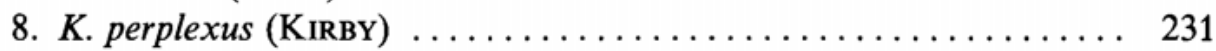

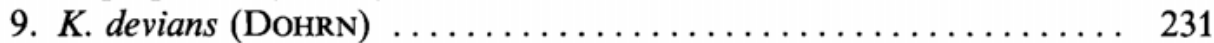

? nigripennis (SCUDDER) $\ldots \ldots \ldots \ldots \ldots \ldots \ldots \ldots \ldots \ldots \ldots \ldots \ldots \ldots \ldots \ldots, 232$

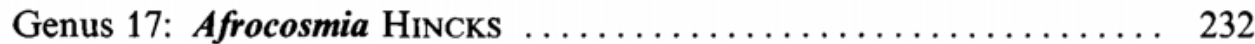

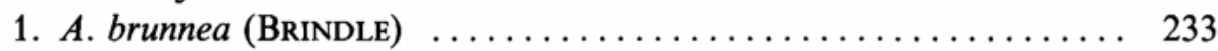

2. A. denticulata HincKs .......................... 234

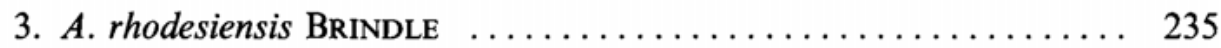

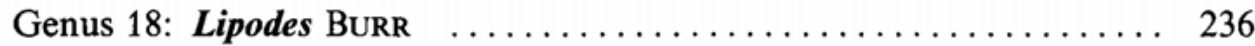

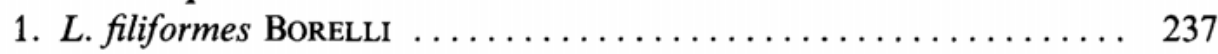

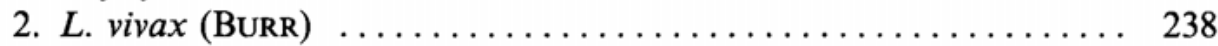

Genus 19: Cosmiola Bey-Bienko ...................... 238

1. C. simplex BeY-Bienko .......................... 239

3. Subfamily Opisthocosmiinae VerHoEfF $\ldots \ldots \ldots \ldots \ldots \ldots \ldots \ldots, 240$

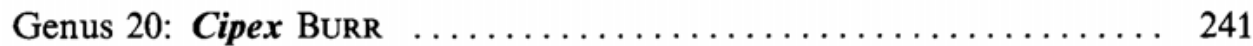

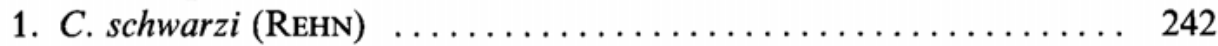

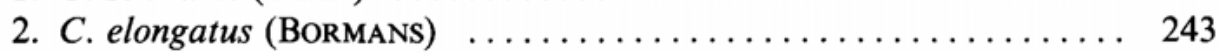

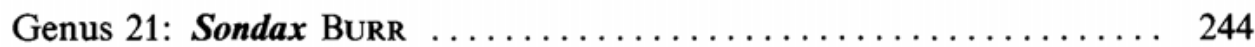

1. S. potanini Bey-BIEnKo ........................ 245

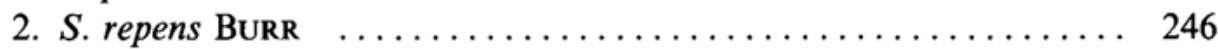

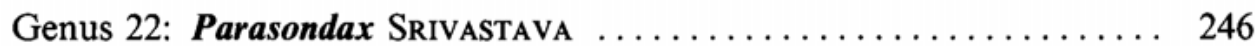

1. P. cantralli SRIVAStava ......................... 247

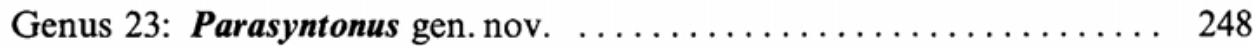

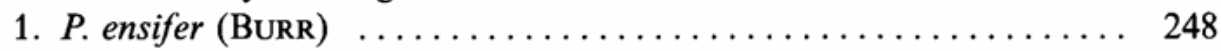




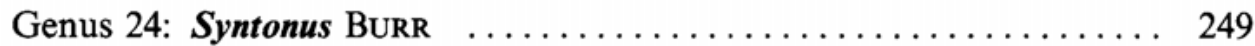

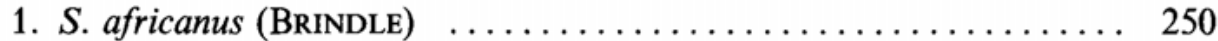

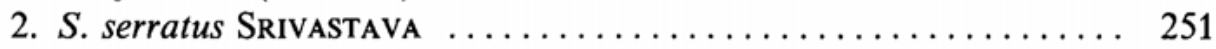

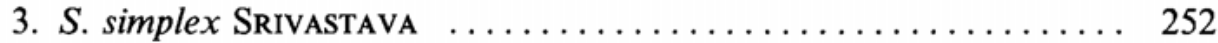

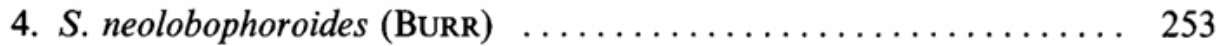

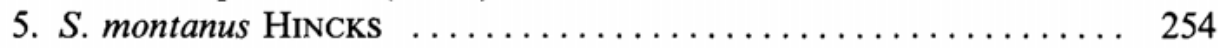

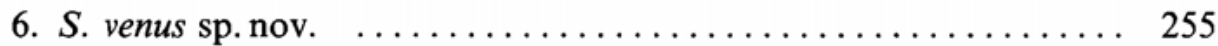

Genus 25: Eutimomena BEY-BIENKo $\ldots \ldots \ldots \ldots \ldots \ldots \ldots \ldots \ldots$

1. E. paradoxa BEY-BIENKO ......................... 256

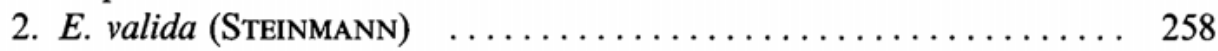

Genus 26: Opisthocosmia DoHRN $\ldots \ldots \ldots \ldots \ldots \ldots \ldots \ldots \ldots \ldots$

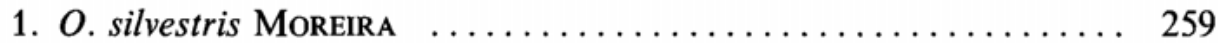

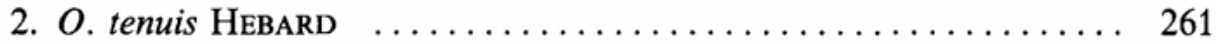

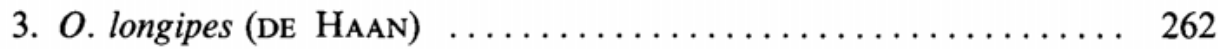

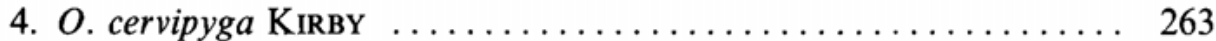

5. O. centurio DoHRN ........................... 264

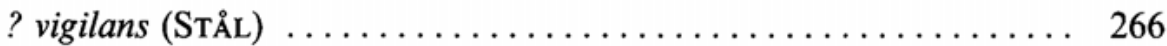

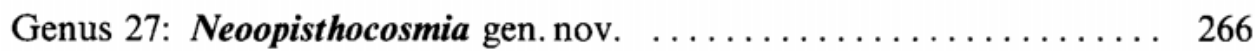

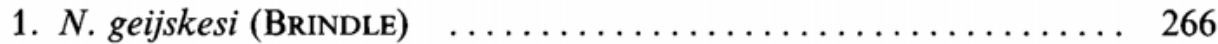

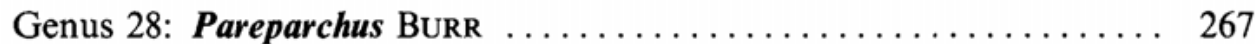

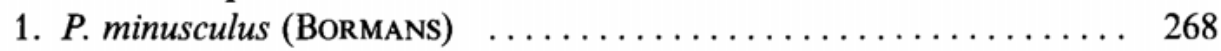

2. P. pelvimeter Hebard ............................ 269

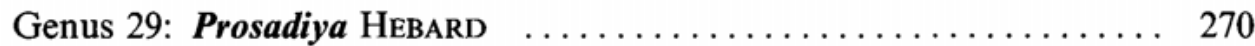

1. P. tricota Hebard .............................. 270

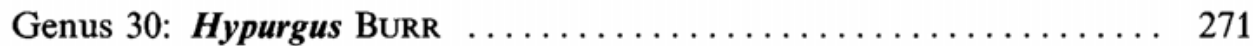

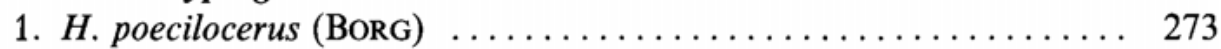

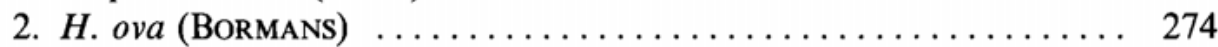

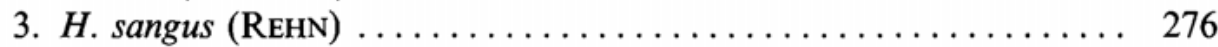

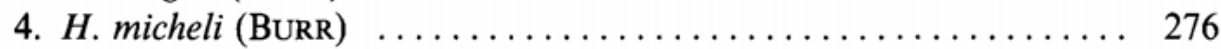

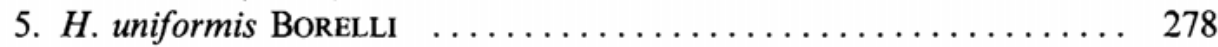

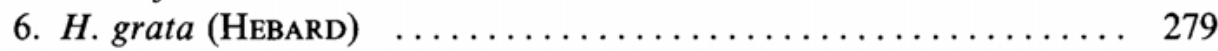

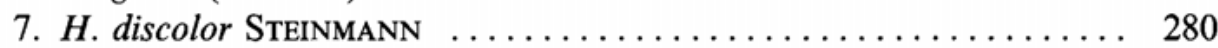

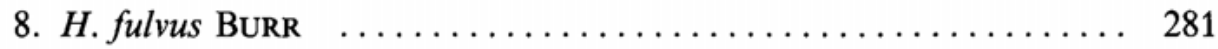

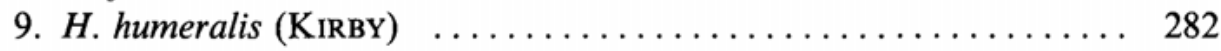

Genus 31: Timomenus BURR $\ldots \ldots \ldots \ldots \ldots \ldots \ldots \ldots \ldots \ldots \ldots \ldots$ 


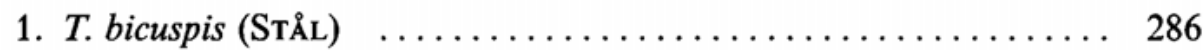

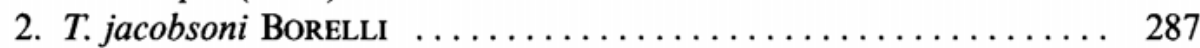

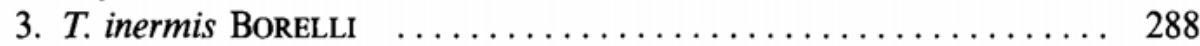

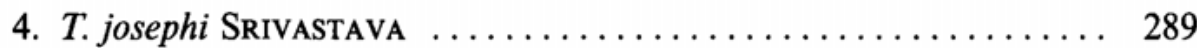

5. T. shelfordi (BURR) _......................... 290

6. T. bicostatus BORELLI .......................... 291

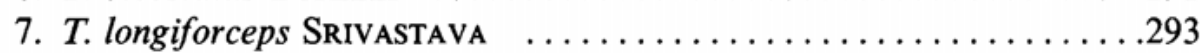

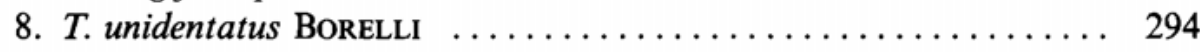

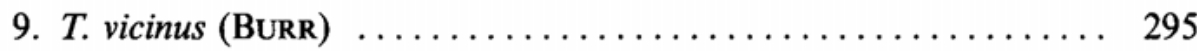

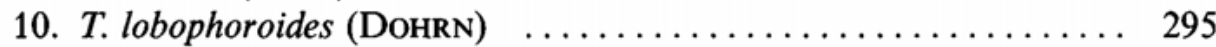

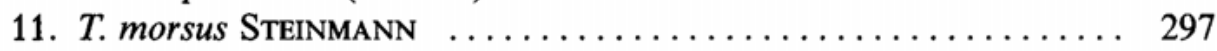

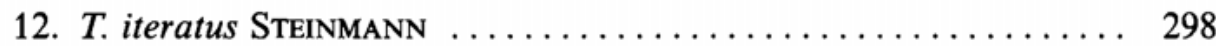

13. T. haddeni SRIVASTAVA .......................... 299

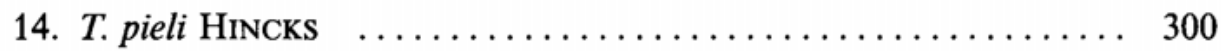

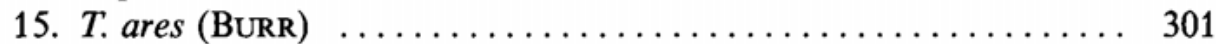

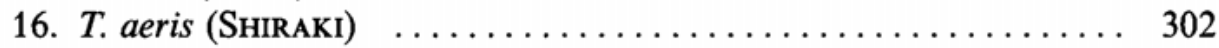

17. T. robustus SRIVASTAVA ...................... 303

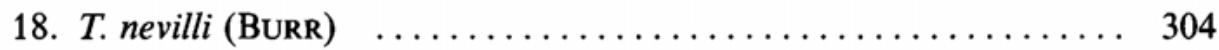

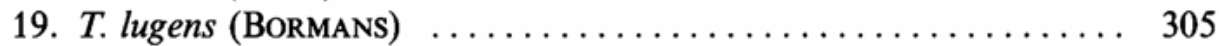

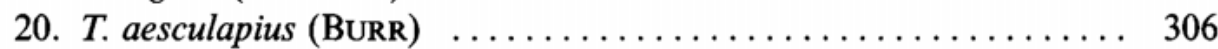

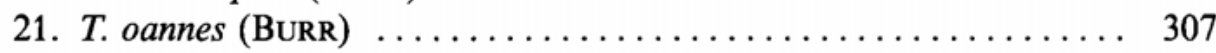

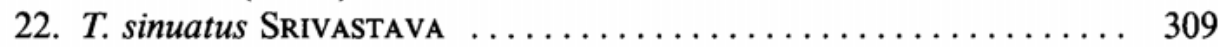

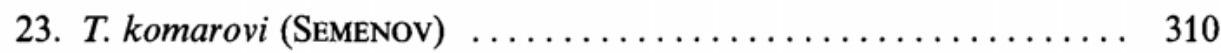

Genus 32: Paratimomenus Steinmann $\ldots \ldots \ldots \ldots \ldots \ldots \ldots \ldots \ldots \ldots$

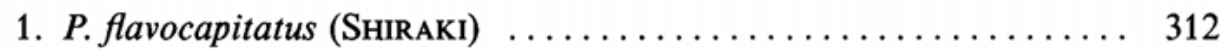

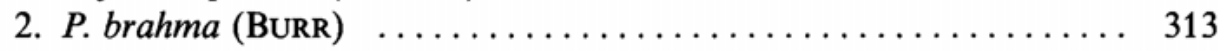

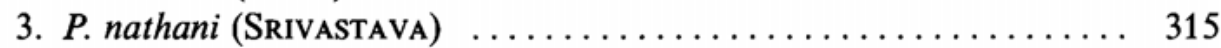

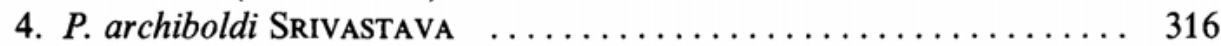

5. P. incognitus sp. nov. ........................... 317

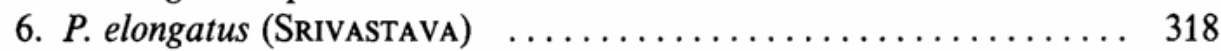

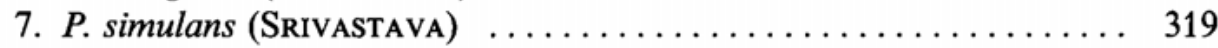

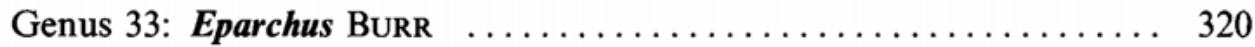

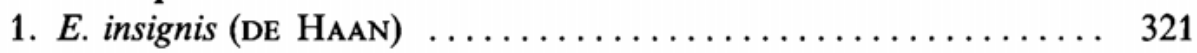

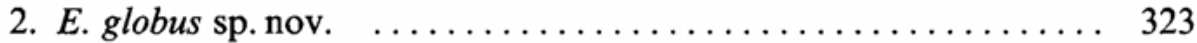

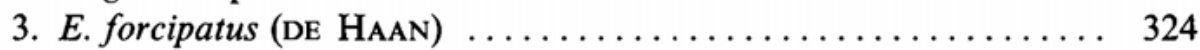

4. E. crucentatus BURR ........................... 325

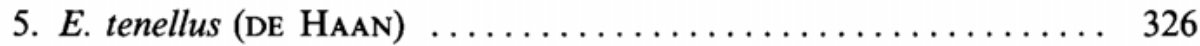

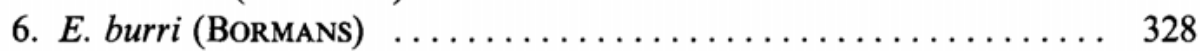

7. E. mindanensis GüNTHER ...................... 329

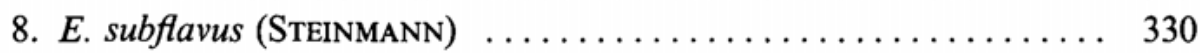




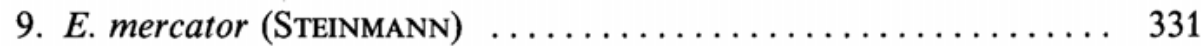

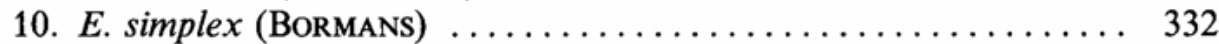

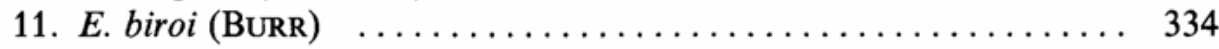

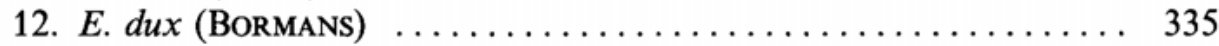

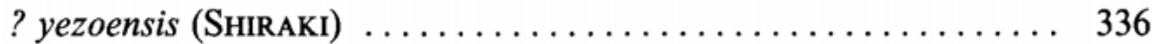

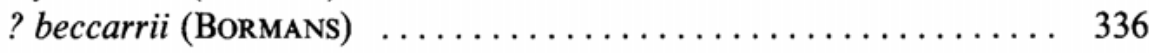

Genus 34: Acanthocordax GüNTHER $\ldots \ldots \ldots \ldots \ldots \ldots \ldots \ldots \ldots \ldots$

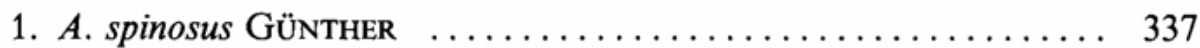

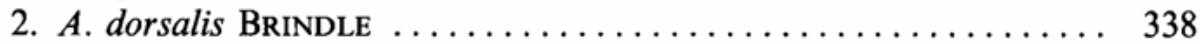

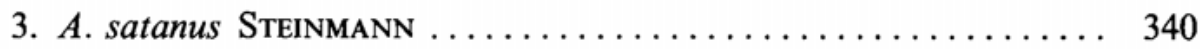

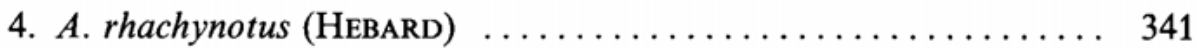

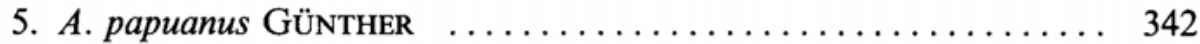

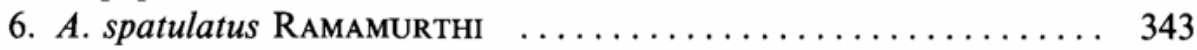

Genus 35: Cordax Burr ............................ 344

1. C. bifurcatus BRINDLE ............................ 345

2. C. formosus (BURR) ........................... 346

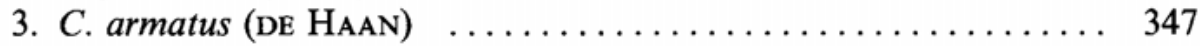

4. C. goliath sp. nov. ............................ 349

5. C. vankampeni BURR ........................ 350

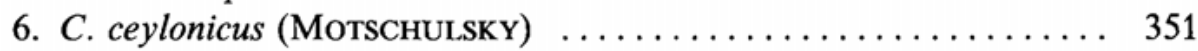

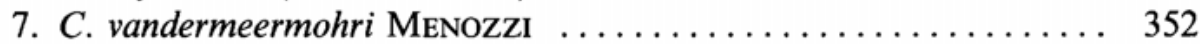

? politus BURR $\ldots \ldots \ldots \ldots \ldots \ldots \ldots \ldots \ldots \ldots \ldots \ldots \ldots \ldots \ldots \ldots$

Genus 36: Spinosocordax Steinmann $\ldots \ldots \ldots \ldots \ldots \ldots \ldots \ldots \ldots . \ldots \ldots 3$

1. S. excelsior SteinMANN $\ldots \ldots \ldots \ldots \ldots \ldots \ldots \ldots \ldots \ldots \ldots \ldots \ldots \ldots \ldots$

Genus ?: Chaetocosmia NishiKawa $\ldots \ldots \ldots \ldots \ldots \ldots \ldots \ldots \ldots \ldots$

? taiwanica NishikaWA ......................... 354

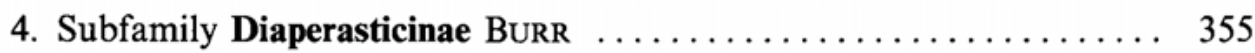

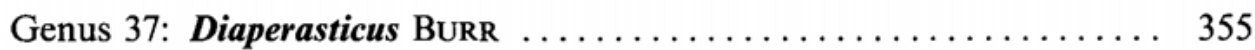

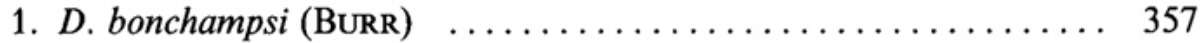

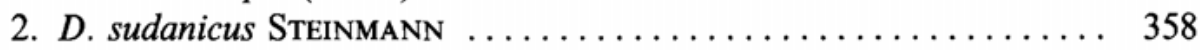

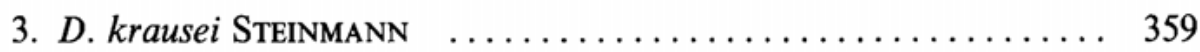

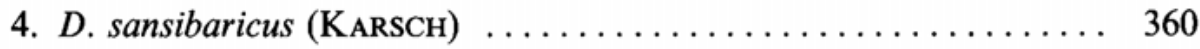

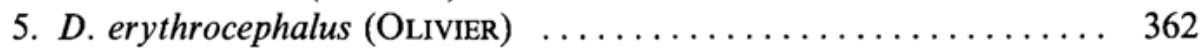

6. D. wittei Hincks .............................. 364

5. Subfamily Allodahlinae VerHOEFF $\ldots \ldots \ldots \ldots \ldots \ldots \ldots \ldots \ldots \ldots$ 


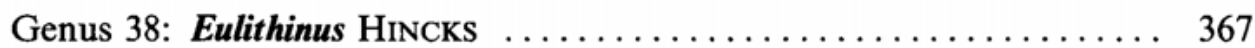

1. E. analis (RAMBUR) .......................... 367

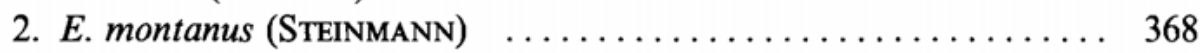

Genus 39: Brindleiana SteInMANN $\ldots \ldots \ldots \ldots \ldots \ldots \ldots \ldots \ldots, 370$

1. B. atlas SteinMANN .......................... 370

Genus 40: Allodahlia VerhoefF $\ldots \ldots \ldots \ldots \ldots \ldots \ldots \ldots \ldots \ldots \ldots \ldots \ldots \ldots$

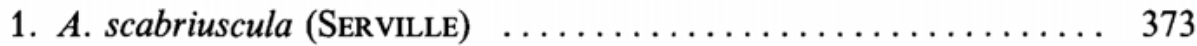

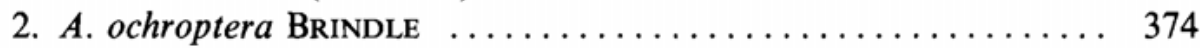

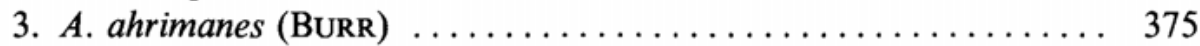

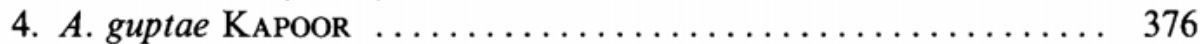

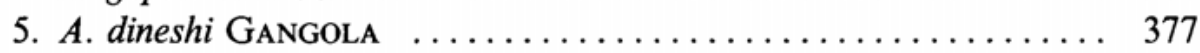

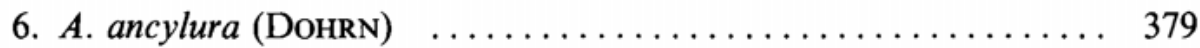

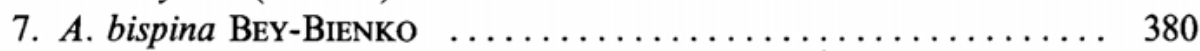

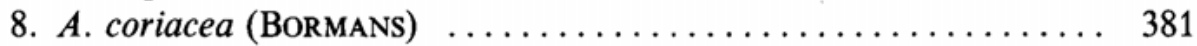

9. A. martensi BRINDLE ......................... 382

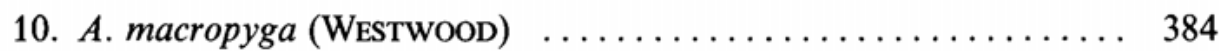

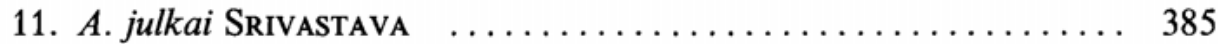

12. A. oxypyga Bey-Bienko ....................... 386

6. Subfamily Anechurinae BURR $\ldots \ldots \ldots \ldots \ldots \ldots \ldots \ldots \ldots \ldots \ldots \ldots \ldots$

Genus 41: Neopterygida Srivastava $\ldots \ldots \ldots \ldots \ldots \ldots \ldots \ldots \ldots$

1. N. circulata (DoHRN) K........................ 390

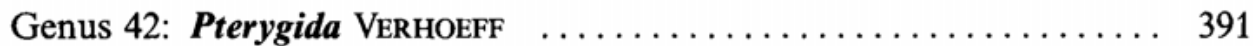

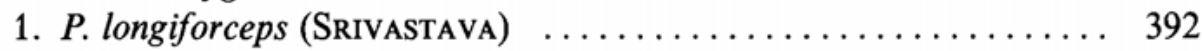

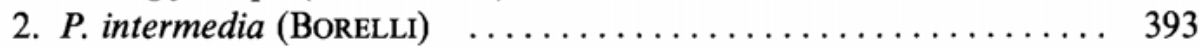

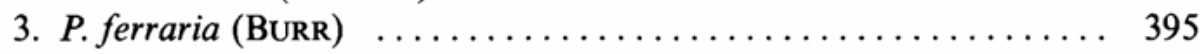

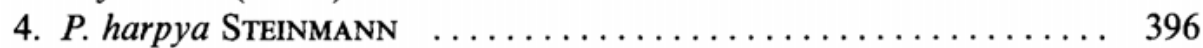

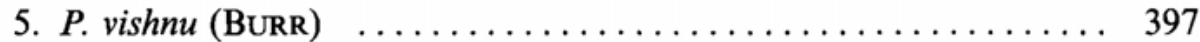

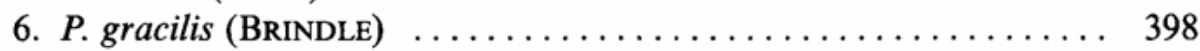

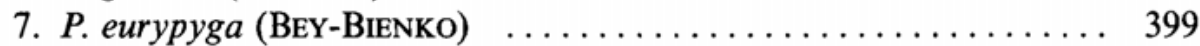

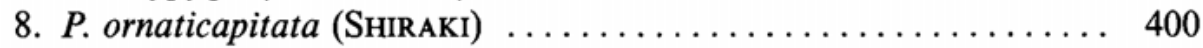

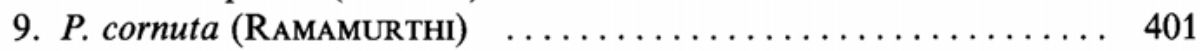

10. P. temora (BURR) ............................... 402

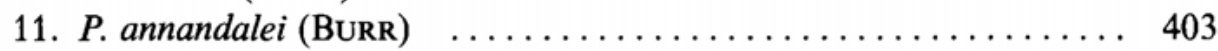

12. P. jagori (DoHRN) ........................... 404

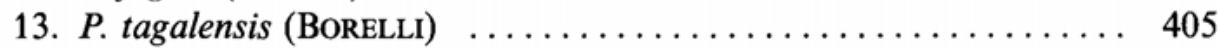

14. P. burgursi (BURR) ........................... 406

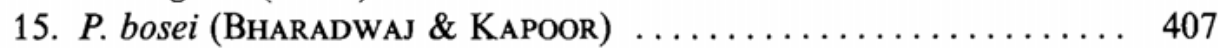

? pulchripes (BoRMANs) ...................... 409 


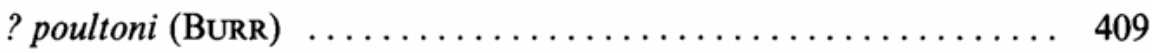

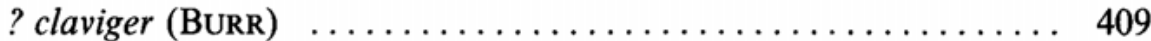

? maculata (BeY-BIENKo) $\ldots \ldots \ldots \ldots \ldots \ldots \ldots \ldots . \ldots . \ldots . \ldots 9$

Genus 43: Chelidura Latreille .......................... 409

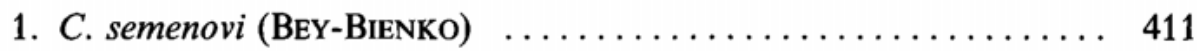

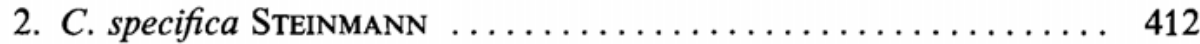

3. C. apfelbecki WeRnER ......................... 413

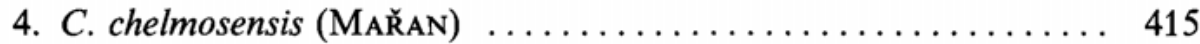

5. C. carpathica (SteinMANN \& Kis) sp. nov. .............. 416

6. C. aptera (Megerle) ........................... 417

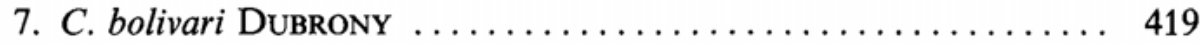

8. C. redux (Semenov) .............................. 420

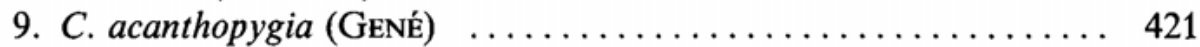

10. C. euxina (Semenov) .......................... 423

11. C. russica (SteinMann) ......................... 424

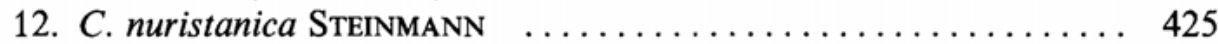

13. C. przewalskii (SEMENov) ........................ 427

14. C. tibetana (Semenov \& Bey-Bienko) $\ldots \ldots \ldots \ldots \ldots \ldots \ldots . \ldots 28$

15. C. mutica Krauss ............................. 429

16. C. pyrenaica (BoNeLLI) ........................ 430

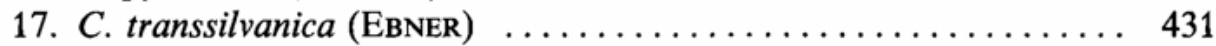

Genus 44: Eumegalura BeY-BIENKo $\ldots \ldots \ldots \ldots \ldots \ldots \ldots \ldots \ldots 433$

1. E. mandarina BEY-BIENKo ....................... 433

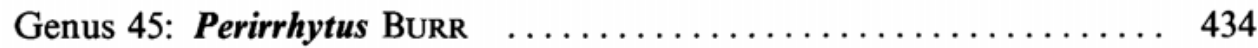

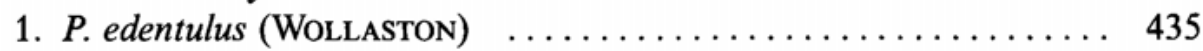

2. P. madeirensis (BoRELLI) ....................... 436

3. P. lundbladi HINCKs ........................... 437

Genus 46: Pseudochelidura VerHOEFF $\ldots \ldots \ldots \ldots \ldots \ldots \ldots \ldots, 438$

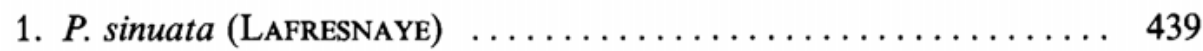

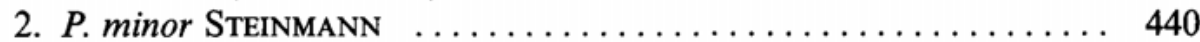

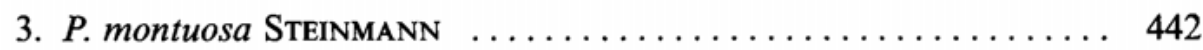

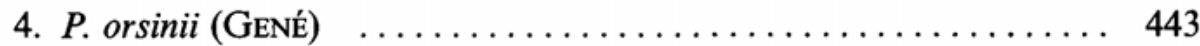

Genus 47: Mesasiobia Semenov ...................... 444

1. $M$. hemixanthocara SemEnov ..................... 445

Genus 48: Oreasiobia SEMENOv $\ldots \ldots \ldots \ldots \ldots \ldots \ldots \ldots \ldots \ldots \ldots 446$

1. O. fedtschenkoi (SAUSSURE) $\ldots \ldots \ldots \ldots \ldots \ldots \ldots \ldots \ldots . \ldots \ldots 47$ 


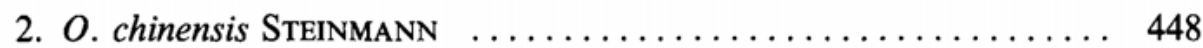

3. O. calciatii (BoRELLI) ......................... 449

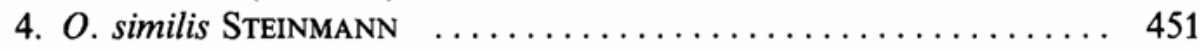

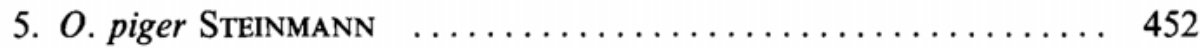

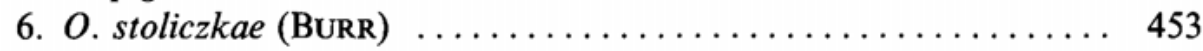

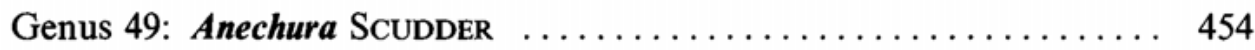

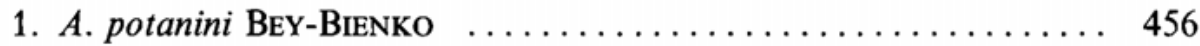

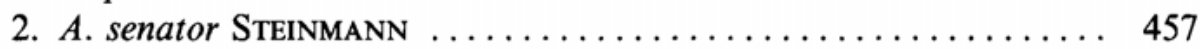

3. A. forficuliformis Semenov \& Bey-Bienko .............. 459

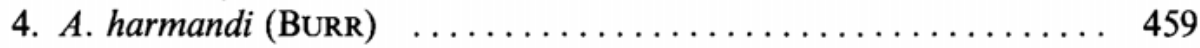

5. A. lewisi (BURR) ............................... 461

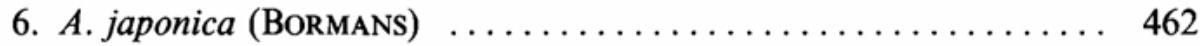

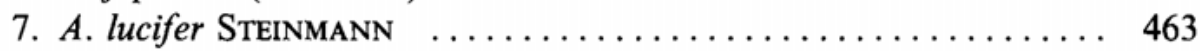

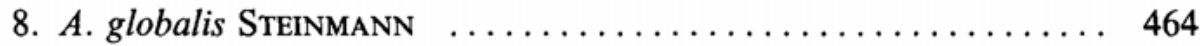

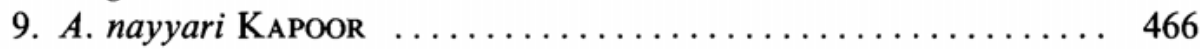

10. A. filchneri (BURR) …........................ 467

11. A. modesta Bey-BIEnKo ........................... 468

12. A. primaria Bey-BIEnKo $\ldots \ldots \ldots \ldots \ldots \ldots \ldots \ldots \ldots \ldots . \ldots \ldots$

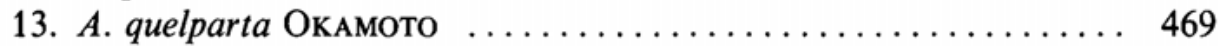

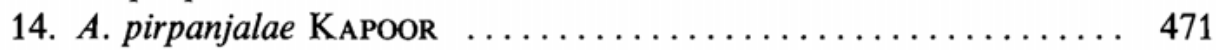

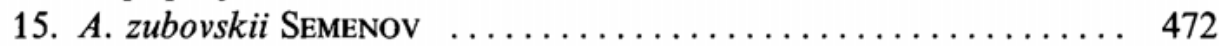

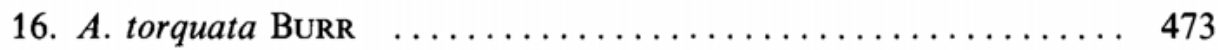

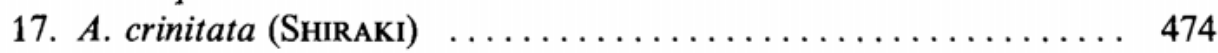

18. A. bipunctata (FABRICIUS) ....................... 475

19. A. svenhedini Bey-BIEnko ...................... 477

? sokotrana BURR ............................ 478

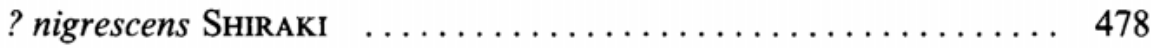

? rubicapitis Liu ............................ 478

7. Subfamily Neolobophorinae BURR $\ldots \ldots \ldots \ldots \ldots \ldots \ldots \ldots \ldots, 478$

Genus 50: Eudohrnia BURR ....................... 479

1. E. metallica (DoHRN) …................... 480

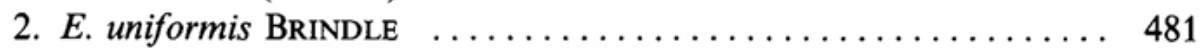

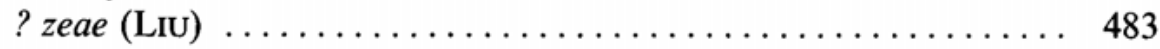

Genus 51: Tristanella Borelli $\ldots \ldots \ldots \ldots \ldots \ldots \ldots \ldots \ldots \ldots \ldots, 483$

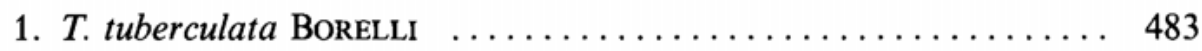

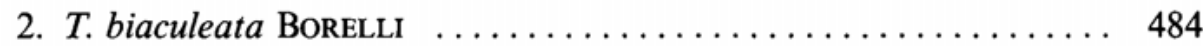

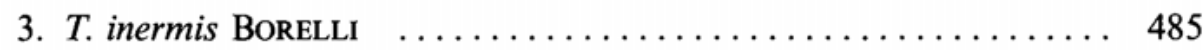


XVIII

Genus 52: Setocordax Brindle $\ldots \ldots \ldots \ldots \ldots \ldots \ldots \ldots \ldots \ldots \ldots$

1. S. gurneyi BRINDLE ......................... 487

Genus 53: Neolobophora SCUDDER $\ldots \ldots \ldots \ldots \ldots \ldots \ldots \ldots \ldots, 488$

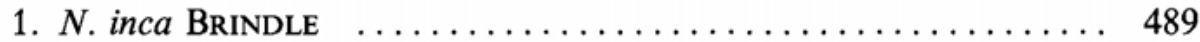

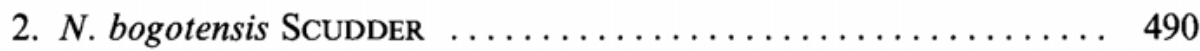

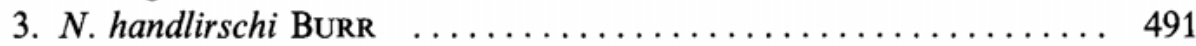

? bicolor BORELLI .......................... 492

Genus 54: Metresura ReHN ..................... 492

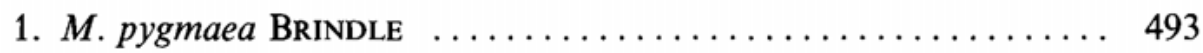

2. M. rugosa BRINDLE ............................ 494

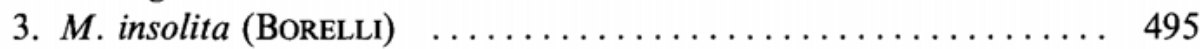

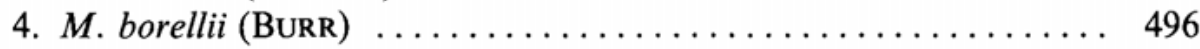

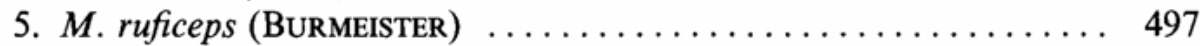

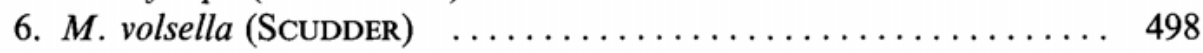

7. M. flaviceps BRINDLE ........................... 499

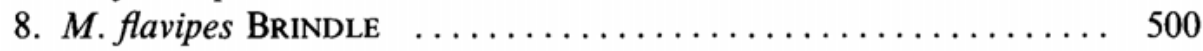

8. Subfamily Forficulinae VerHOEFF $\ldots \ldots \ldots \ldots \ldots \ldots \ldots \ldots \ldots \ldots$

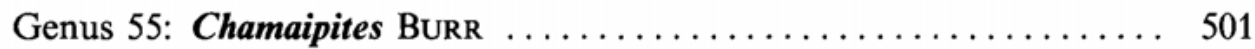

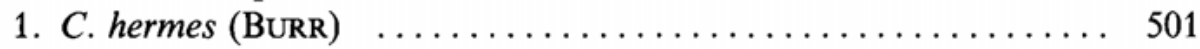

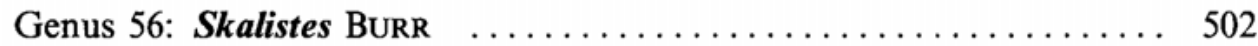

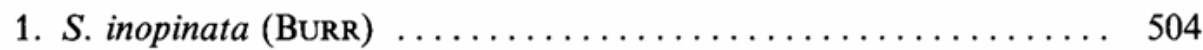

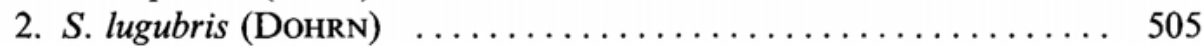

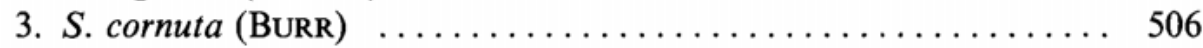

4. S. cacaoensis (CAUDELL) ......................... 507

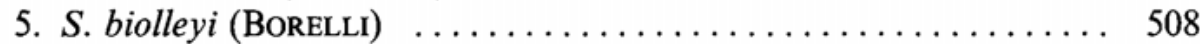

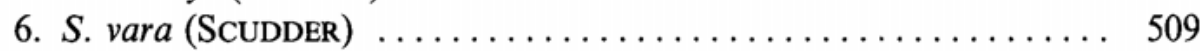

7. S. lucasi BRINDLE ............................ 510

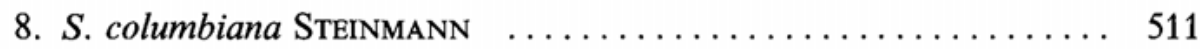

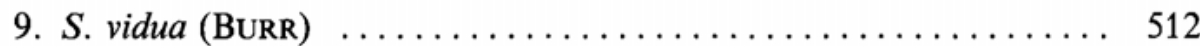

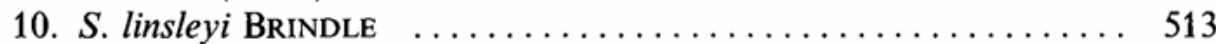

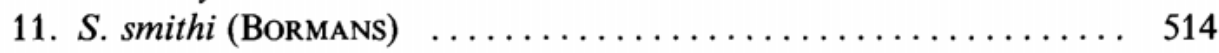

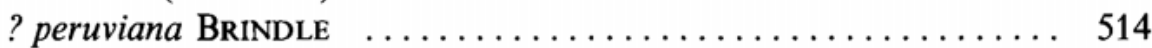

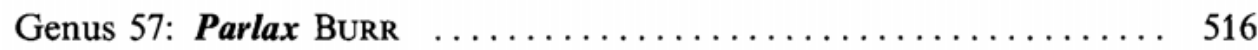

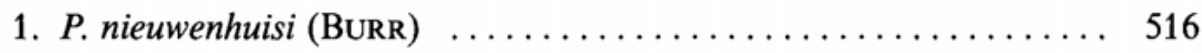

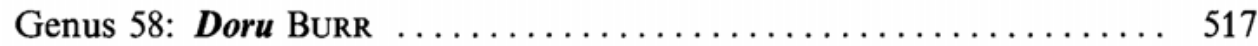

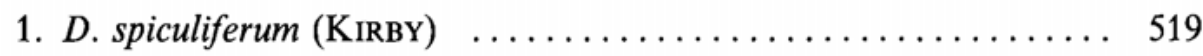




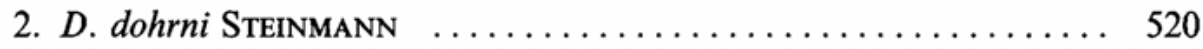

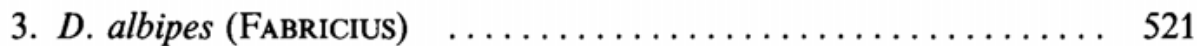

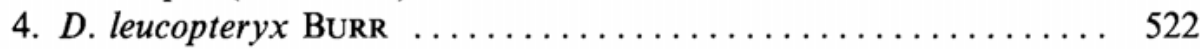

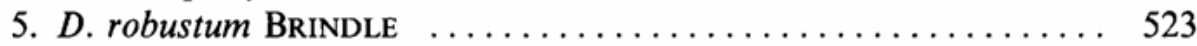

6. D. platensis Borelli ......................... 524

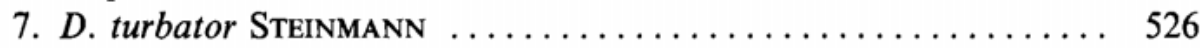

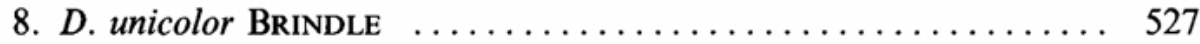

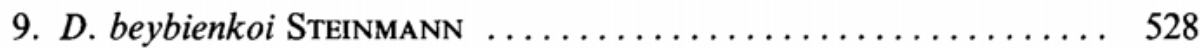

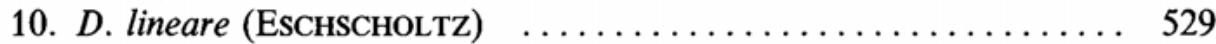

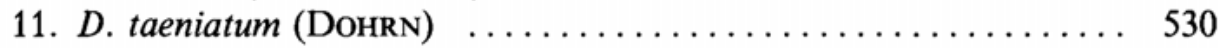

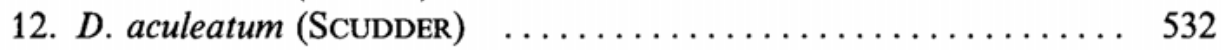

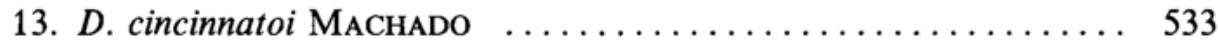

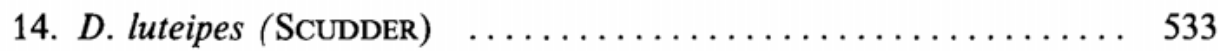

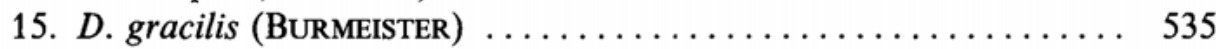

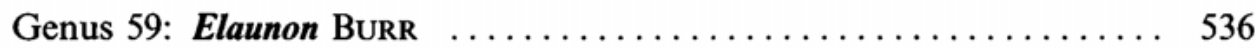

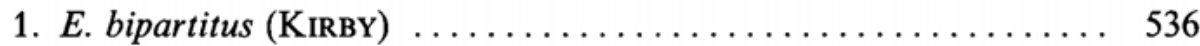

? gangoli Gangola $\ldots \ldots \ldots \ldots \ldots \ldots \ldots \ldots \ldots \ldots \ldots \ldots \ldots \ldots \ldots$

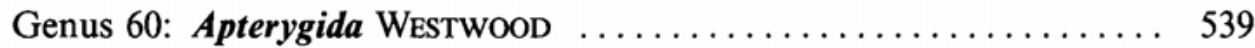

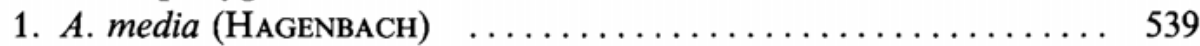

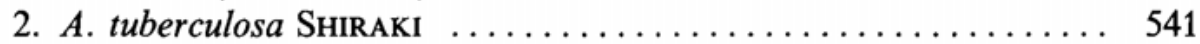

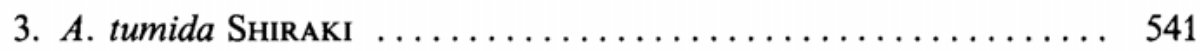

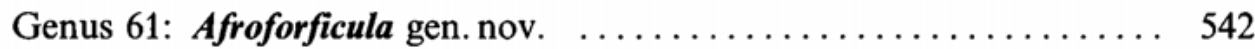

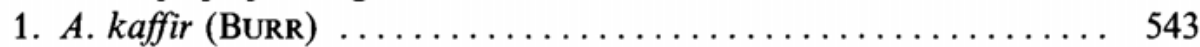

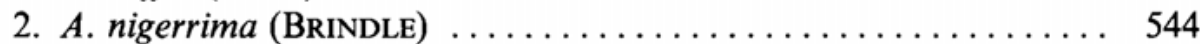

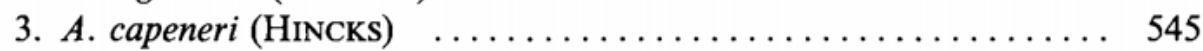

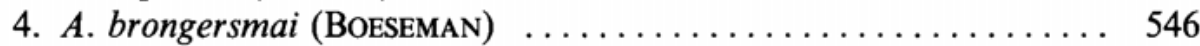

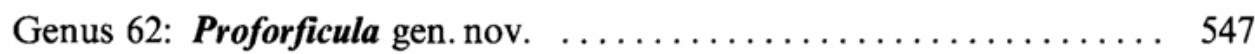

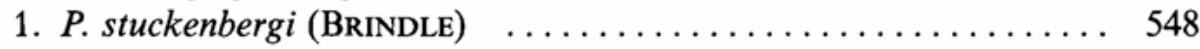

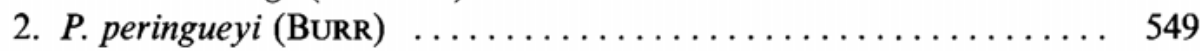

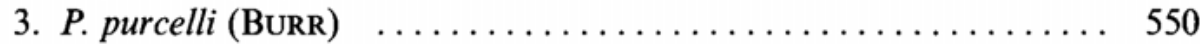

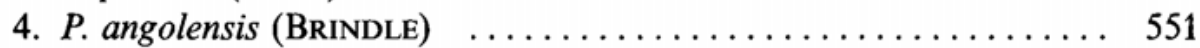

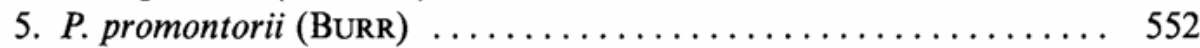

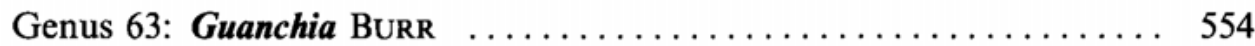

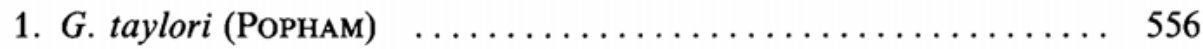

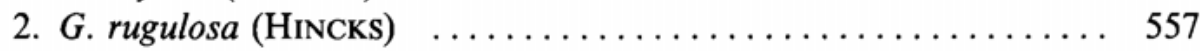

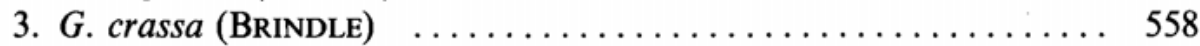

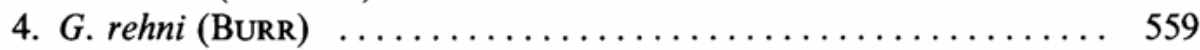




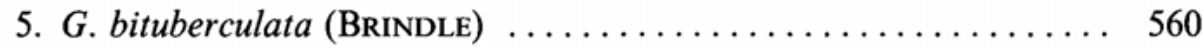

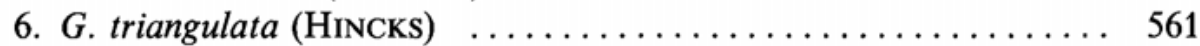

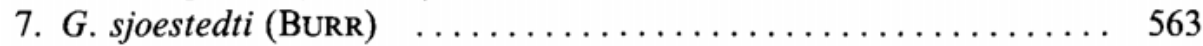

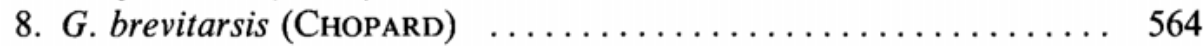

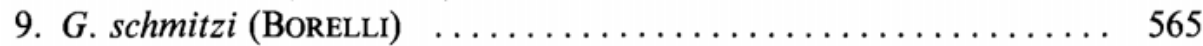

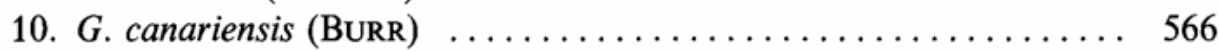

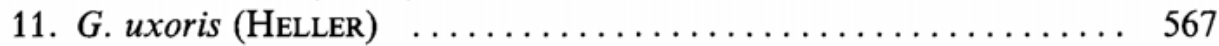

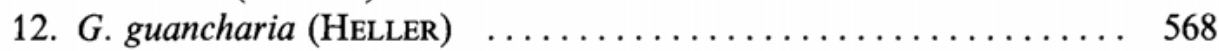

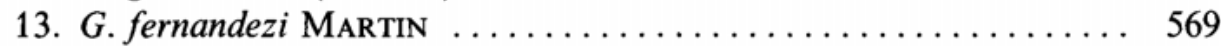

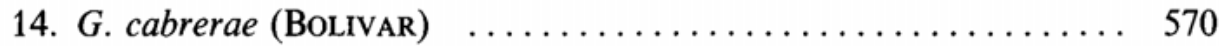

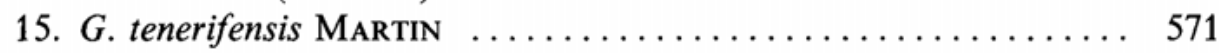

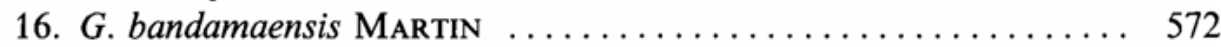

17. G. transversa BRINDLE ......................... 573

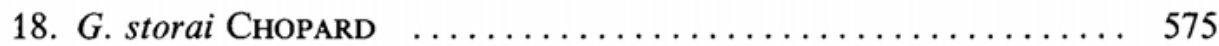

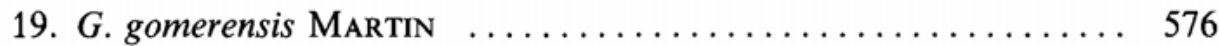

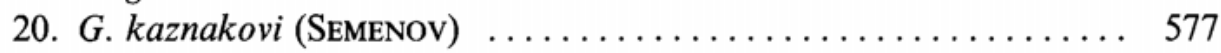

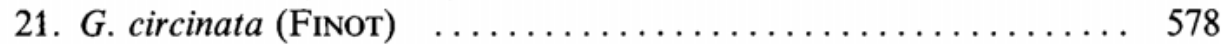

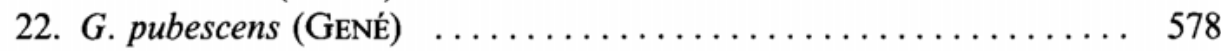

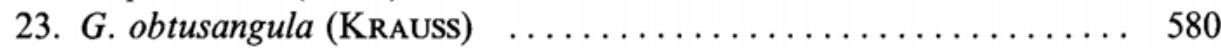

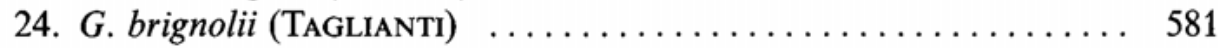

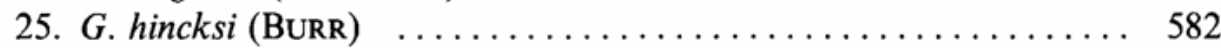

26. G. medica BURR ............................ 583

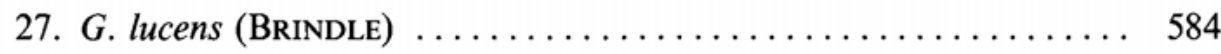

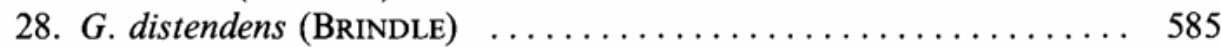

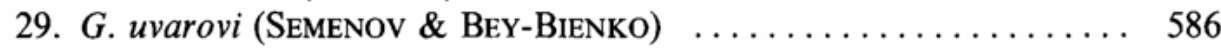

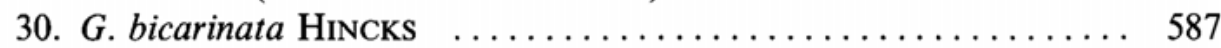

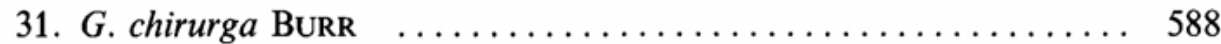

Genus 64: Forficula Linnaeus $\ldots \ldots \ldots \ldots \ldots \ldots \ldots \ldots \ldots \ldots \ldots$

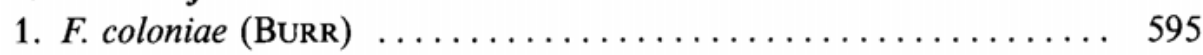

2. F. chopardi (HINCKS) .......................... 596

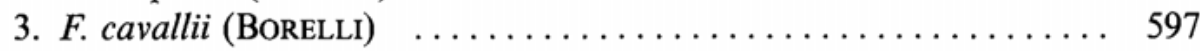

4. F. picta KIRBY ................................ 598

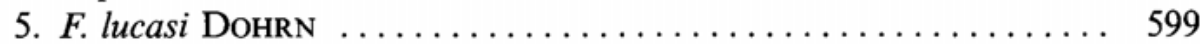

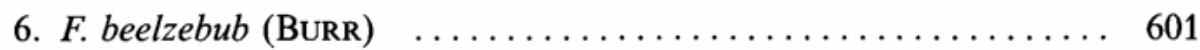

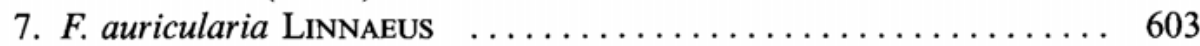

8. F. borellii BRINDLE ............................. 605

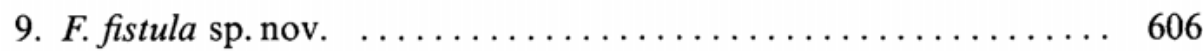

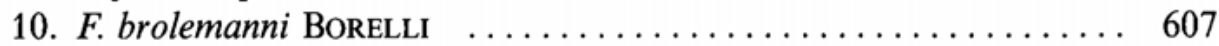

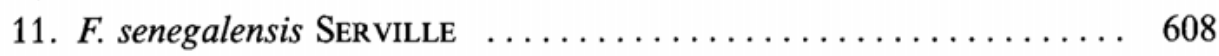

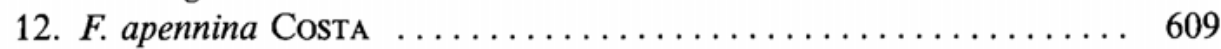

13. F. lesnei Finot ............................... 611 


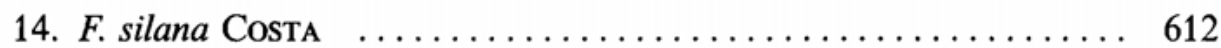

15. F. aetolica BRUNNER ............................. 613

16. F. decipiens GeNÉ .............................. 614

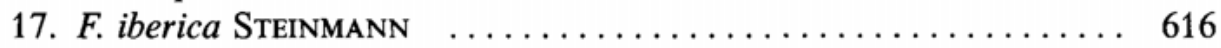

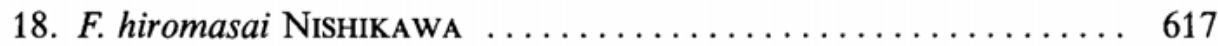

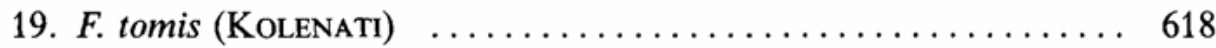

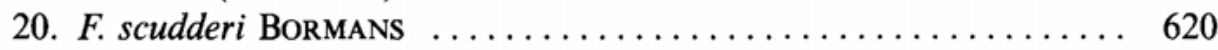

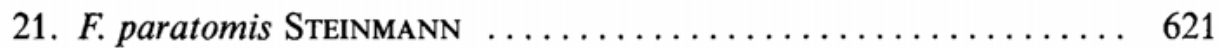

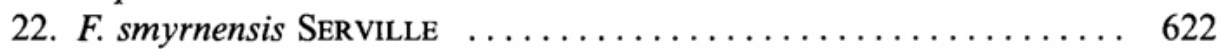

23. F. mikado BURR .............................. 624

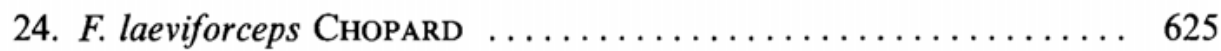

25. F. abrutiana BoRelli ........................... 626

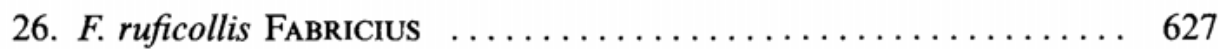

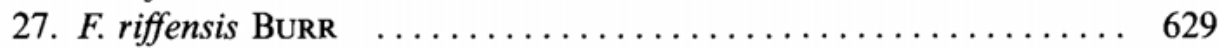

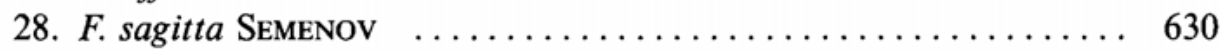

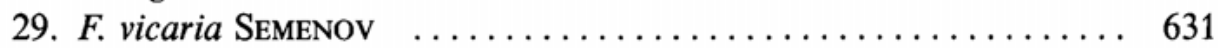

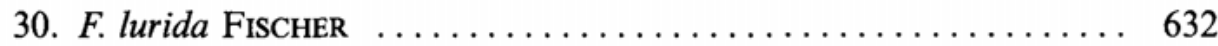

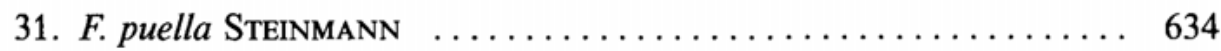

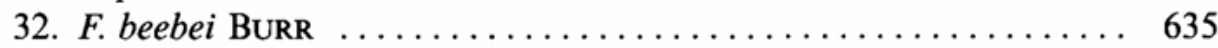

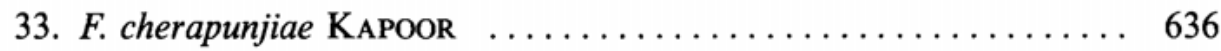

34. F. kashmirensis SRIVASTAVA ..................... 637

35. F. kambaitensis HincKs ........................ 638

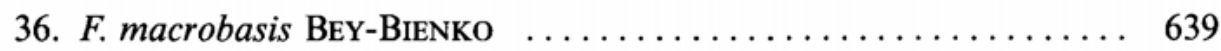

37. F. externa BEY-BIENKo ........................... 641

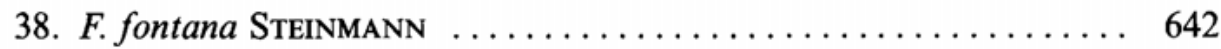

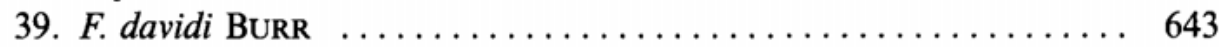

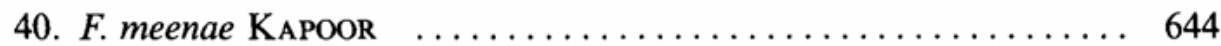

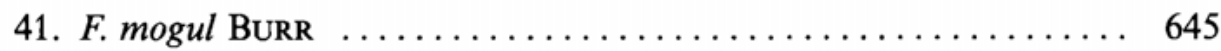

42. F. subauricularia BeY-Bienko ...................... 646

43. F. berezovskyi BEY-BIENKo ...................... 647

44. F. sinica Bey-BIENKo ................................ 648

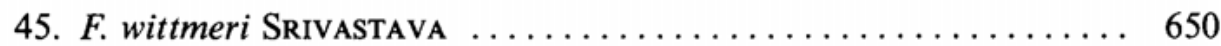

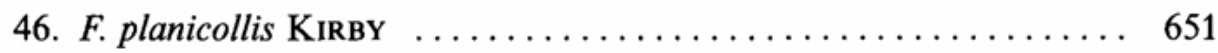

47. F. cristata SRIVASTAVA ........................ 652

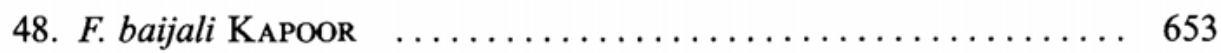

49. F. biplaga BeY-BienKo ........................... 655

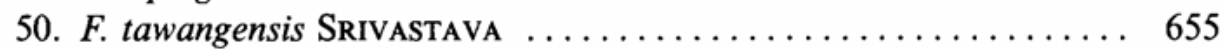

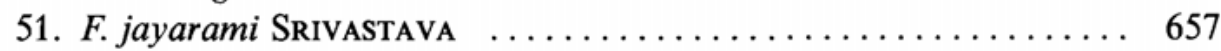

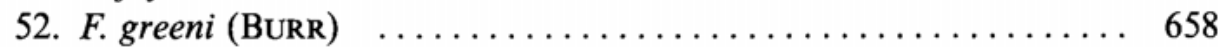

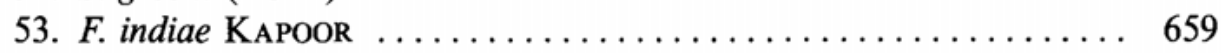

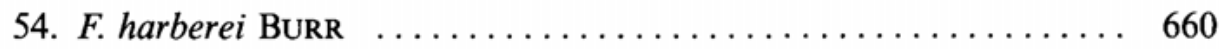

55. F. abbottabadiensis BHARADWAJ \& KAPOOR ............... 661 


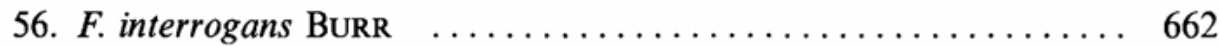

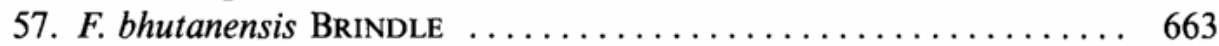

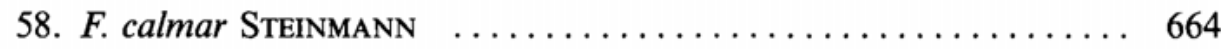

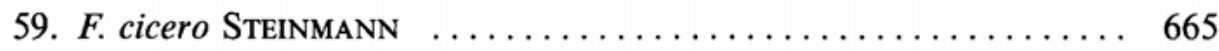

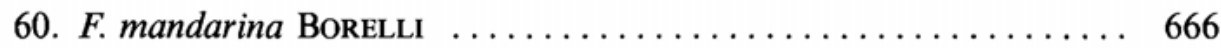

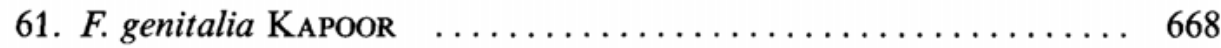

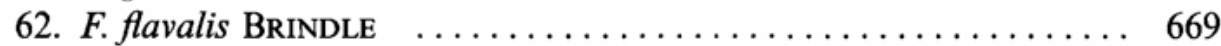

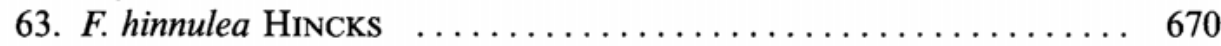

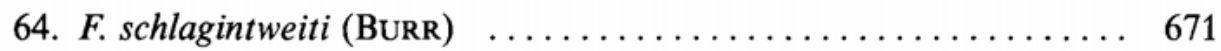

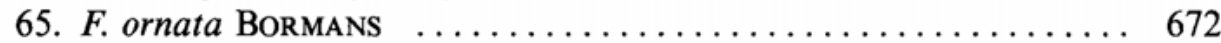

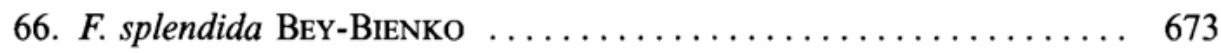

67. F. vilmi SteinMANN .............................. 674

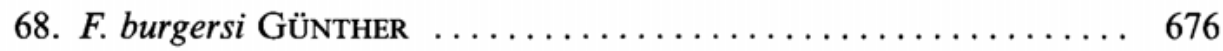

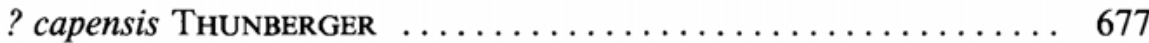

? mabillei RochENBERG . .......................... 677

? flexuosa FABRICIUS ........................... 677

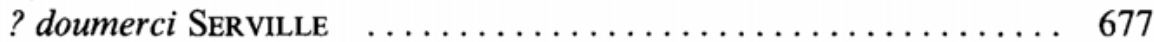

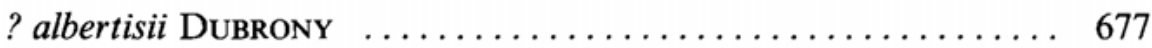

? gravelyi BURR .............................. 677

? kinfomontis LIU ............................. 677

? fasciata THUNBERger $\ldots \ldots \ldots \ldots \ldots \ldots \ldots \ldots \ldots \ldots \ldots$

Genus ?: Mesolabia SHIRAKI _........................... 678

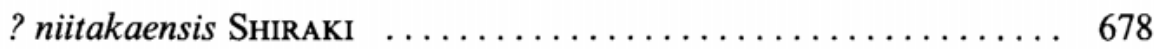

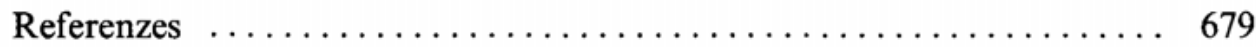

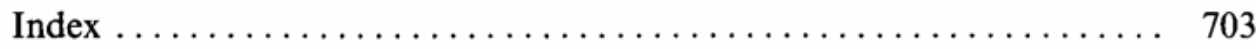

